\title{
Radio/FADS/IMU Integrated Navigation for Mars Entry
}

\author{
Xiuqiang Jiang ${ }^{\mathrm{a}, \mathrm{b} \dagger}$, Shuang $\mathrm{Li}^{\mathrm{a}, \mathrm{b} \ddagger}$ and Xiangyu Huang ${ }^{ }$ \\ a. College of Astronautics, Nanjing University of Aeronautics and Astronautics, Nanjing 210016, China \\ b. Laboratory of Space New Technology, Nanjing University of Aeronautics and Astronautics, Nanjing 210016, China \\ c. Beijing Institute of Control Engineering, Beijing 100190, China
}

\begin{abstract}
Supposing future China's orbiting and landing collaborative exploration mission as the potential project background, this paper addresses the issue of Mars entry integrated navigation using radio beacon, flush air data sensing system (FADS), and inertial measurement unit (IMU). The range and Doppler information sensed from an orbiting radio beacon, the dynamic pressure and heating data sensed from flush air data sensing system, and acceleration and attitude angular rate outputs from an inertial measurement unit are integrated in an unscented kalman filter to perform state estimation and suppress the system and measurement noise. Computer simulations show that the proposed integrated navigation scheme can enhance the navigation accuracy, which enables precise entry guidance for the given Mars orbiting and landing collaborative exploration mission.
\end{abstract}

Keywords: Mars entry, integrated navigation, radio measurement, flush air data sensing system, inertial measurement unit, unscented kalman filter

\section{Introduction}

In order to collect more comprehensive first-hand data and obtain more scientific returns, orbiting and landing collaborative mission has been becoming one of the most attractive Mars exploration modes. To date, only the 1976 Viking Mars landing mission simultaneously conducted orbiting and landing exploration, but the test of radio communication between the orbiter and the entry vehicle was not considered [1]. Typical orbit-alone exploration missions, such as Mars Odyssey (MO), Mars Reconnaissance Orbiter (MRO), and Mangalyaan were launched in 2001, 2005, and 2013, respectively [2, 3]. For landing-alone and surface exploration missions, such as 1996 Mars Pathfinder, the cruise vehicle is not required to participate in subsequent exploration activities after separation [4]. The ultra-high frequency (UHF) band radio communication between the entry vehicle and several available orbiters

\footnotetext{
${ }^{\dagger}$ Ph.D. candidate, Department of Astronautics Engineering; also visiting research scholar in Space Systems Engineering Lab, University of Arizona, Tucson, Arizona 85721; E-mail: jiangxq@nuaa.edu.cn.

‡ Corresponding author, Professor, Department of Astronautics Engineering, E-mail: lishuang@nuaa.edu.cn.

${ }^{\S}$ Professor, Department of Control Engineering.
} 
(such as MO and MRO) during Mars entry phase has been successfully verified by the 2004 Mars Exploration Rover (i.e. Spirit and Opportunity), 2008 Phoenix, and 2012 Mars Science Laboratory (MSL) missions [5-7]. ExoMars, a new orbiting and landing collaborative mission, orbited the red planet in September 2016 [8]. Compared with traditional Mars exploration missions, the orbiting/landing collaborative mission has the following remarkable characteristics: 1) the Mars probe consists of an orbiter and an entry vehicle, and the entry vehicle usually contains a lander and/or a rover; 2) the orbiter conducts the global survey mission, and the lander/rover carries out the pinpoint landing and local surface exploration mission; 3) they (i.e. orbiter, lander and/or rover) support each other through radio communication during their whole mission-cycle [9]. The orbiting/landing collaborative mission provides an excellent opportunity for the orbiter(s) to support entry navigation, which is conductive to improve the entry navigation accuracy using radio measurement.

Mars entry phase is of vital importance for the whole Mars mission-cycle, which begins when the vehicle reaches Mars atmosphere interface and ends at deployment of supersonic parachute. According to the references [9-11], the entry interface is usually defined as a point $3522 \mathrm{~km}$ from the center of Mars, and the reference equator radius of Mars is $3397 \mathrm{~km}$. The entry interface point is usually defined as 125km above the Mars Orbiter Laser Altimeter (MOLA) elevation. In order to achieve a smaller terminal state error and further reduce landing error ellipse, the closed-loop guidance navigation and control (GNC) system with high-precision navigation and guidance performance is needed to guide the entry vehicle through the hypersonic phase to the supersonic flight phase and reach the pre-designed parachute deployment site within a desired deviation [10-12]. China has already initiated the technical preparation for its own Mars orbiting/landing collaborative exploration missions, including the advanced navigation and guidance technologies for Mars atmospheric entry. To date, all Mars entry vehicles continue to rely on the inertial measurement unit (IMU) based dead reckoning navigation mode developed in the Viking missions [11-13]. The navigation performance usually degrades with time due to the inertial constant biases and drifts from IMU, and the position error of Mars entry is approximately a few kilometers [12, 13].

To improve the navigation accuracy of Mars entry, one feasible solution is utilizing the external measurements to correct the inertial biases and drifts. However, most external sensors, except for UHF radio beacons, cannot be used before the heat shield is automatically separated because there is a heat shield to protect the entry vehicle from adverse aerodynamic heating environment $[6,7,11,12]$. Recently, the radio/IMU integrated navigation scheme for Mars entry has been widely addressed using different orbiting/surface radio beacons and filter algorithms [12-19]. Previous studies show that the accuracy of radio/IMU integrated navigation is significantly better than that of traditional inertial navigation when both the configuration of multiple beacons and observability meet certain 
conditions. Unfortunately, the accuracy of radio/IMU integrated navigation is hardly upgraded when only one radio beacon is available [20-22]. Hence, the alone radio/IMU integrated navigation only using one radio beacon is not a feasible solution to support high-precision entry guidance of the first Mars orbiting/landing collaborative exploration mission. In the 2012 MSL mission, the entry vehicle carried a set of instruments in-situ measuring the pressure and temperature distribution on the aeroshell. The instrumentation is what we called flush air data sensing system (FADS). It consists of Mars entry atmospheric data system (MEADS) to take the pressure measurements and Mars integrated sensor plug (MISP) to take the aerothermodynamic data [23-25]. The original purpose of FADS is to confirm the new aerodynamic design and further perfect Mars entry dynamics knowledge. The data from both FADS and IMU has been used to reconstruct Mars entry profile and atmosphere, and the reconstruction accuracy is better than that of only based on the outputs of IMU [25-27]. From the navigation point of view, the FADS can also be considered as a new external measurement to support Mars entry navigation. However, the alone FADS/IMU integrated estimation scheme cannot effectively restrain the divergence of position error [25-27].

The aim of this paper is to extend the preceding work reported in [9,14-16] and numerically verify radio/FADS/IMU integrated navigation schemes for Mars entry phase, which is expected to be a feasible solution to support the future Chinese Mars orbiting/landing collaborative exploration mission. In this integrated navigation framework, both the relative range and velocity information from the entry vehicle to the orbiter sensed by radio measurement and the dynamic pressure and temperature data sensed by the FADS are added as new external navigation measurements to improve traditional inertial navigation. The rest of this paper is organized as follows. Section 2 conceptually describes a candidate Mars orbiting/landing collaborative exploration mission and entry integrated navigation scheme. Section 3 introduces the three degree-of-freedom (3-DOF) and 6-DOF Mars entry dynamical model adopted in the subsequent sections of this paper respectively. Section 4 defines navigation measurement models utilized in the integrated navigation algorithm. In Section 5, Radio/FADS/IMU integrated navigation algorithm is presented at length, and the observability is discussed accordingly. In Section 6, the composition of the simulation system is described, and simulation results are discussed in detail. Finally, Section 7 contains conclusion.

\section{Integrated Entry Navigation Scheme for Mars Orbiting/Landing Collaborative Mission}

China's first Mars exploration mission utilizes the orbiting/landing collaborative exploration mode, which is conductive to obtain more engineering data and accumulate more technical foundations for subsequent Mars exploration activities [9]. Meanwhile, more available sensors data and observable measurements are also conductive 
to improve the traditional navigation accuracy of Mars entry. The given Mars orbiting/landing collaborative mission case employed aerodynamics sensors and UHF-band radio orbiter-to-spacecraft telemetry. In other words, available navigation sensors for Mars entry phase include IMU, radio and FADS. Therefore, radio/FADS/IMU integrated navigation is a possible solution in this case in order to improve the navigation accuracy of Mars entry. Fig.1 depicts the schematic diagram of Radio/FADS/IMU integrated navigation for the entry phase of the Mars orbiting/landing collaborative exploration mission. According to the past engineering experiences, the estimated position error and velocity error are respectively expected to be less than $1 \mathrm{~km}$ and $20 \mathrm{~m} / \mathrm{s}$ for atmospheric entry phase [12].

The past Mars telecommunication relay tests showed that radio range and velocity measurement can be obtained through UHF band radio communication between the orbiter and entry vehicle, which can be used for Mars entry navigation $[7,13,14]$. As a mature technology, radio measurement has been widely used to provide navigation services for various aerospace vehicles in China. As only one pair of range and Doppler information is sensed in the Mars orbiting/landing collaborative mission, an optimal geometric configuration between the orbiter and the entry vehicle is required to be properly selected in advance from the navigation observability point of view to ensure good navigation performance. Because the entry vehicle separates from the orbiter thirty minutes before the beginning of Mars entry, the orbiter should precisely maneuver to its optimal orbit after separation. Note that the optimal configuration of orbiting beacons based on navigation observability was well studied in reference [21] and [22], so it will not be discussed anymore in this paper.

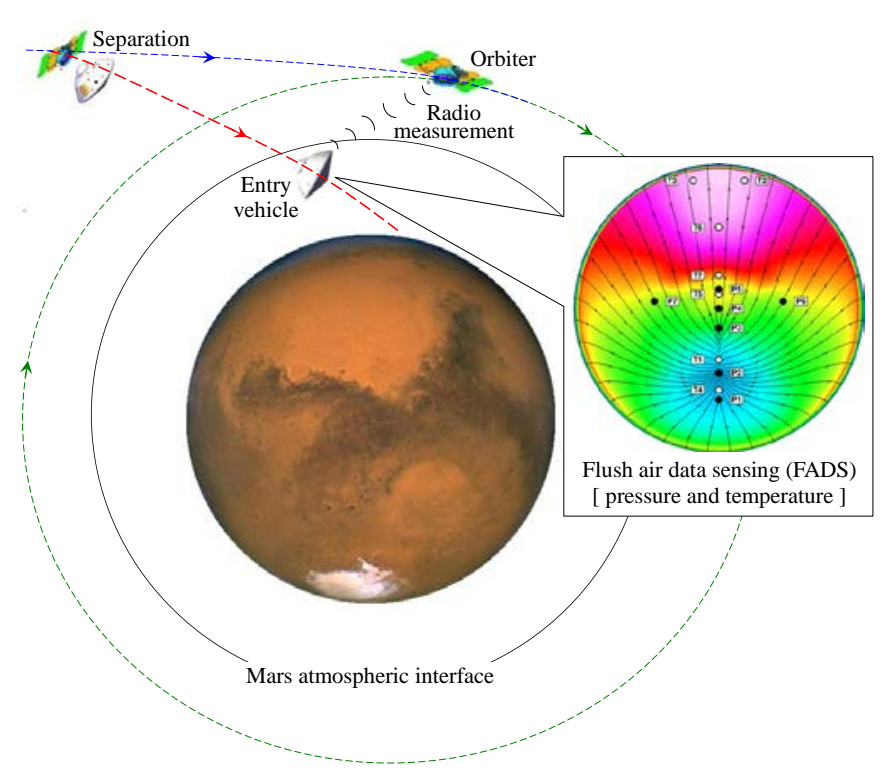

Fig. 1. Schematic diagram of Radio/FADS/IMU integrated navigation for Mars orbiting/landing collaborative mission [9].

FADS technologies have been widely developed and utilized for various aerospace vehicles. The FADS 
developed for planetary entry vehicle consists of pressure transducers and thermal plugs to respectively sense the dynamic pressure and heating on the fore-body of the vehicle. They are embedded in the thermal protection system (TPS) [23-25]. According to the entry vehicle design and preplanned flight phase, the numbers, type, and installation location of FADS sensors are required to be optimized by aerodynamicist in advance. According to the measured pressure and heating data, the entry vehicle's velocity, angle-of-attack, sideslip angle, as well as atmospheric density can be identified through their specific mathematical relations [25-27]. The identification process does not completely depend on the outputs of IMU and Mars atmosphere model. Thus, the measurement data from FADS is introduced as a new external measurement.

\section{Mars Entry Dynamical Model}

In order to develop the real Mars entry dynamics for simulation and provide system state equations for navigation filter, the 3-DOF and 6-DOF dynamic equations of Mars entry are constructed in this section respectively.

\subsection{3-DOF Dynamic Equations of Mars Entry}

Mars atmospheric entry is a hypersonic flight process, and lasts a very short period of time. Therefore, the MSL-type low-lifting entry vehicle can be considered as an unpowered point mass flying in a stationary atmosphere, and Mars is assumed to be a regular spheroid with uniformly distributed mass. The entry vehicle is commanded to fly at a constant trim angle of attack, and the entry guidance of the vehicle is achieved only through adjusting the bank angle by use of a reaction control system (RCS). The 3-DOF dynamic equations of Mars entry vehicle, defined with respect to the Mars centered Mars-fixed coordinate system, are given by [12, 14, and 21]

$$
\left\{\begin{array}{l}
\&=v \sin \gamma \\
\&=\frac{v \cos \gamma \sin \psi}{r \cos \lambda} \\
\&=\frac{v \cos \gamma \cos \psi}{r} \\
\&=-D-g(r) \sin \gamma \\
\&=\left(\frac{v}{r}-\frac{g(r)}{v}\right) \cos \gamma+\frac{L \cos \sigma}{v} \\
\&=\frac{v \sin \psi \cos \gamma \tan \lambda}{r}+\frac{L \sin \sigma}{v \cos \gamma}
\end{array}\right.
$$

where $r$ denotes the distance between the center of Mars and the center of mass of entry vehicle, $\theta$ and $\lambda$ are longitude and latitude respectively, $v$ stands for the velocity of entry vehicle, $\gamma$ is the flight path angle. $\psi$ is the heading angle, with $\psi=0$ as due north. As the only control variable for entry phase, bank angle $\sigma$ is defined as the angle about the velocity vector from the local vertical plane to the lift vector.

Mars gravitational acceleration is defined by 


$$
g(r)=\mu / r^{2}
$$

where $\mu$ represents Mars gravitational constant.

$D$ and $L$ denote the aerodynamic drag and lift accelerations respectively, defined by

$$
\left\{\begin{array}{l}
D=q B \\
L=D \cdot(L / D)
\end{array}\right.
$$

where dynamic pressure $q=\frac{1}{2} \rho(h) v^{2}$, ballistic coefficient $B=C_{D} S / m$, lift-drag ratio $(L / D)=C_{L} / C_{D} . C_{D}$ and $C_{L}$ denote the aerodynamic drag and lift coefficients respectively. $S$ is the vehicle reference surface area, and $m$ is the mass of entry vehicle. According to NASA's Mars global reference atmospheric model (Mars-GRAM) derived from data fitting, the Mars atmospheric density model utilized in this paper is given as follows $[12,16]$

$$
\rho(h)=\rho_{0} \exp (\beta(h))
$$

where $\rho_{0}=559.351005946503 / a T, \quad a=188.95110711075, \quad T=1.4 \times 10^{-13} h^{3}-8.85 \times 10^{-9} h^{2}-1.245 \times 10^{-3} h+205.3645$, $\beta(h)=-0.000105 h$, Mars is assumed to be a perfect sphere with the surface radius $r_{0}$, and altitude $h=r-r_{0}$. The unit of atmospheric density is $\mathrm{kg} / \mathrm{m}^{3}$, and the unit of altitude is $\mathrm{m}$.

\subsection{6-DOF Dynamic Equations of Mars Entry}

The entry vehicle is flying under the effect of aerodynamic force and gravity. The Mars entry trajectory can be adjusted by regulating lift vector, which is realized through controlling bank angle of the entry vehicle. The bank angle is usually considered as the only control variable to simplify the control algorithm design. Traditional 3-DOF dynamic model defined in Eq. (1) only represents the Mars entry translational dynamics without including attitude kinematics. In order to completely describe the system state variables of the entry vehicle, the attitude kinematics should be included into Mars entry dynamic equations. The 6-DOF Mars entry dynamic equations in the Mars centered inertial coordinate system can be formulated as follows [12, 15, and 16]

$$
\left\{\begin{array}{l}
\&=\boldsymbol{v} \\
\&=\boldsymbol{T}_{V}^{I} \boldsymbol{a}_{V}+\boldsymbol{T}_{G}^{I} \boldsymbol{g}_{G} \\
\boldsymbol{\&}=\boldsymbol{K} \boldsymbol{\omega}_{B}
\end{array}\right.
$$

where $\boldsymbol{r}=\left[r_{x}, r_{y}, r_{z}\right]^{\mathrm{T}}$ denotes the position vector from the center of Mars to the center of mass of entry vehicle, $\boldsymbol{v}=\left[v_{x}, v_{y}, v_{z}\right]^{\mathrm{T}}$ denotes the velocity vector of the entry vehicle, $\boldsymbol{\Omega}=[\varphi, \vartheta, \phi]^{\mathrm{T}}$ denotes the tri-axial attitude angle, $\boldsymbol{\omega}_{B}=\left[\omega_{x}, \omega_{y}, \omega_{z}\right]^{\mathrm{T}}$ is the real tri-axial angular rate described in the vehicle body-fixed coordinate system. $\boldsymbol{a}_{V}$ is the aerodynamic acceleration described in the velocity coordinate system, $\boldsymbol{g}_{G}$ is the Mars gravitational 
acceleration described in the Mars centered geographic coordinate system, $\boldsymbol{K}$ is the attitude kinematics matrix. $\boldsymbol{a}_{V}, \boldsymbol{g}_{G}$ and $\boldsymbol{K}$ are respectively defined as follows $[12,15,16]$

$$
\begin{gathered}
\boldsymbol{a}_{V}=[-D,-L \sin \sigma, L \cos \sigma]^{\mathrm{T}} \\
\boldsymbol{g}_{G}=\left[0,0,-\frac{\mu}{\|\boldsymbol{r}\|^{2}}\right]^{\mathrm{T}} \\
\boldsymbol{K}=\frac{1}{\cos \vartheta}\left[\begin{array}{ccc}
\cos \vartheta & \sin \vartheta \sin \varphi & \sin \vartheta \cos \varphi \\
0 & \cos \vartheta \cos \varphi & -\cos \vartheta \sin \varphi \\
0 & \sin \varphi & \cos \varphi
\end{array}\right]
\end{gathered}
$$

Note that $\boldsymbol{T}_{V}^{I}$ is the coordinate transformation matrix from the velocity coordinate system to the Mars centered inertial coordinate system, and $\boldsymbol{T}_{G}^{I}$ is the coordinate transformation matrix from the geographic coordinate system to the Mars centered inertial coordinate system. Both of the coordinate system definitions and coordinate transformation matrixes can been easily found in our previous works [14-16].

\section{Navigation Measurement Models}

The measurements adopted in the integrated navigation system include the outputs form on-board IMU, FADS and the radio measurement between the entry vehicle and the orbiter. The navigation measurement models used for subsequent navigation filter are described at length in this section.

\subsection{IMU Measurement Model}

IMU suit includes a number of accelerometers and gyros, which are usually used to sense linear acceleration along three orthogonal axes and rotation rates around three orthogonal axes. The IMU measurement model is represented as follows [12, 14]

$$
\begin{aligned}
& d_{B}=\boldsymbol{a}_{B}+\boldsymbol{b}_{a}+\boldsymbol{\xi}_{a} \\
& \boldsymbol{d}_{B}=\omega_{B}+\boldsymbol{b}_{\omega}+\boldsymbol{\xi}_{\omega}
\end{aligned}
$$

where $\boldsymbol{d}_{B}$ and $\boldsymbol{\phi}_{\mathrm{B}}$ are the measured acceleration and angular velocity data from accelerometers and gyros respectively, $\boldsymbol{b}_{a}$ and $\boldsymbol{b}_{\omega}$ are the acceleration bias and angular rate bias respectively, $\boldsymbol{\xi}_{a}$ and $\boldsymbol{\xi}_{\omega}$ are the acceleration output noise and angular rate output noise respectively (assumed to be white Gaussian noise). $\boldsymbol{a}_{B}$ and $\boldsymbol{\omega}_{B}$ are the real acceleration and angular velocity data respectively. $\boldsymbol{a}_{B}$ is constructed as $\boldsymbol{a}_{B}=\boldsymbol{T}_{V}^{B} \boldsymbol{a}_{V}, \boldsymbol{a}_{V}$ is defined in Eq. (6). $\boldsymbol{T}_{V}^{B}$ is the coordinate transformation matrixes from the velocity coordinate system to the body-fixed coordinate system, which can be found in our previous works [14-16]. 
Then, the IMU measurement model can be constructed as follows

$$
\boldsymbol{y}_{I M U}=\left[\begin{array}{l}
\boldsymbol{d}_{\mathrm{B}} \\
\boldsymbol{\partial} \theta_{\mathrm{B}}
\end{array}\right]=\left[\begin{array}{l}
\boldsymbol{T}_{V}^{B} \boldsymbol{a}_{V} \\
\boldsymbol{\omega}_{B}
\end{array}\right]+\left[\begin{array}{l}
\boldsymbol{b}_{a}+\boldsymbol{\xi}_{a} \\
\boldsymbol{b}_{\omega}+\boldsymbol{\xi}_{\omega}
\end{array}\right]
$$

As the IMU outputs are considered as navigation measurements in this paper, the acceleration bias and angular rate bias are treated as measurement noises.

\subsection{FADS Measurement Model}

The dynamic pressure and heating data around the forebody of the entry vehicle can be sensed using FADS distributed multiple sensors. Advanced FADS developed for planetary reentry/entry vehicles is expected to output the magnitude of dynamic pressure, the unit vector along the direction of dynamic pressure in the body-fixed coordinate system, and aerodynamic heating [23-25, 29]. Especially, the unit vector along the direction of velocity is collinearly reverse relative to the unit vector along the direction of dynamic pressure when the entry vehicle stably flies at a constant trim angle of attack, so the unit vector along the direction of velocity in the body-fixed coordinate system can be obtained. Consequently, the FADS has the ability to support measuring and estimating of aerodynamic parameters, which include atmospheric density, sound velocity, Mach number, drag coefficient and lift coefficient. At the same time, the outputs from FADS can also be used for measuring and estimating of flight states, such as velocity, angle of attack, and sideslip angle[23-28]. And also, a satisfactory measurement accuracy of the FADS can be reached through optimizing configuration and calibrating precisely [25]. Note that the value of velocity relative to Martian atmosphere is assumed to be the same as value of velocity in the Mars centered inertial coordinate system when Martian atmosphere is assumed to be stationary during the hypersonic entry process.

The real magnitude of dynamic pressure $q$, the unit vector in the direction of dynamic pressure $\boldsymbol{q}_{e}$, and the temperature rate $\&$ at the stagnation point are respectively defined as follows [29,31]

$$
\begin{gathered}
q=\frac{1}{2} \rho(h) v^{2} \\
\mathcal{F}_{=}=\frac{\&}{C} \\
\&=k_{q}\left(\frac{\rho(h)}{r_{n}}\right)^{N} v^{M} \\
\boldsymbol{q}_{e}=-v_{e}
\end{gathered}
$$

where $\&$ is the aerodynamic heating rate, $v_{e}$ is the unit vector in the direction of velocity described in the body-fixed coordinate system, the heat capacity coefficient $C=350 \mathrm{~J} / \mathrm{m}^{2} \mathrm{~K}$, the vehicle nose radius $r_{n}=0.66 \mathrm{~m}$, and 
planet-dependent constants are $k_{q}=1.9027 \times 10^{-4} \sqrt{\mathrm{kg}} / \mathrm{m}, N=0.5, M=3$ [26,29-31]. In fact, Mars atmospheric density is usually difficult to accurately model in advance. According to Eq.(12) to Eq.(15), Mars atmospheric density can be estimated using the velocity data from the outputs of IMU [25, 26]. In the same way, the estimated velocity from integrated navigation is utilized to estimate Mars atmospheric density in this paper. According to the coordinate transformation matrix $\boldsymbol{T}_{v}^{B}$ from the velocity coordinate system to the body-fixed coordinate system, $\alpha_{0}$ is trim angle of attack, the unit vector in the velocity direction $v_{e}$ can be easily constructed as follows [29,30]

$$
\boldsymbol{v}_{e}=\frac{\boldsymbol{v}}{\|\boldsymbol{v}\|}=\left[\cos \alpha_{0},-\sin \alpha_{0} \cos \sigma,-\sin \alpha_{0} \sin \sigma\right]^{\mathrm{T}}
$$

Measurement noise must be taken into account when a navigation measurement model is set up. Then, the FADS measurement model can be constructed as follows

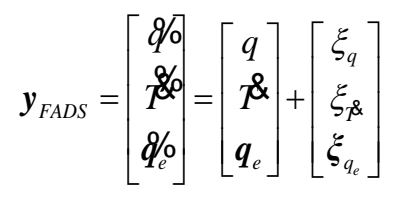

where $8,01 \%, 8,0$ denote the measured dynamic pressure, temperature rate at the stagnation point, and the unit vector in the direction of velocity respectively, $\xi_{q}, \xi_{z} \xi_{q_{e}}$ represent their measurement noises respectively.

It should be pointed out that the measurement noises of FADS in the case of extremely rarefied atmosphere (i.e. entry interface and initial stage) and approximately sonic speed (i.e. entry terminal stage) are larger than that under the conditions of hyper- and supersonic speed and denser atmosphere. Here, these measurement noises are assumed to be white Gaussian noise for verifying the effectiveness of the proposed navigation scheme theoretically.

\subsection{Radio Measurement Model}

The UHF band radio communication between the entry vehicle and the orbiter can be used to improve the accuracy of the integrated navigation for Mars entry in the orbiting/landing collaborative mission. The Radio sensors detect the range and Doppler information through identifying the signal delay, phase and frequency offset in the communication signal carrier. In practice, on-board radio measurement is realized using two-way range radio and two-way Doppler radio. The two-way range will take range measurement from the entry vehicle to the orbiter, and the two-way Doppler will take velocity measurement from the entry vehicle to the orbiter [12, 14].

The radio measurements aforementioned are closely related to the position and velocity of the orbiter. As the whole process of Mars entry only lasts a very short time (e.g. $\sim 4$ min for MSL entry phase), the perturbation influence on the orbit of the orbiter can be negligible. Thus, the position and velocity of the orbiter can be obtained in the Mars centered inertial coordinate system according to the two-body orbit dynamics. The state equations of 
the orbiter are defined as follows [12, 14]

$$
\begin{gathered}
\boldsymbol{v}_{0}=\boldsymbol{v}_{o} \\
\mathscr{\&}_{0}=-\frac{\mu}{\left\|\boldsymbol{r}_{o}\right\|^{3}} \boldsymbol{r}_{o}
\end{gathered}
$$

The real relative range $R$ and velocity $V$ between the entry vehicle and the orbiter are constructed as follows

$$
\begin{aligned}
& R=\sqrt{\left(\boldsymbol{r}-\boldsymbol{r}_{0}\right)^{\mathrm{T}}\left(\boldsymbol{r}-\boldsymbol{r}_{0}\right)} \\
& V=\left(\boldsymbol{r}-\boldsymbol{r}_{0}\right)^{\mathrm{T}}\left(\boldsymbol{v}-\boldsymbol{v}_{0}\right) / R
\end{aligned}
$$

where $\boldsymbol{r}$ and $\boldsymbol{v}$ respectively denote position vector and velocity vector from the center of Mars to the center of mass of entry vehicle in the Mars centered inertial coordinate system.

Then, radio measurement model can be constructed as follows [12, 14-16]

$$
\boldsymbol{y}_{\text {Radio }}=\left[\begin{array}{l}
R \\
V / 9
\end{array}\right]=\left[\begin{array}{l}
R \\
V
\end{array}\right]+\left[\begin{array}{l}
\xi_{R} \\
\xi_{V}
\end{array}\right]
$$

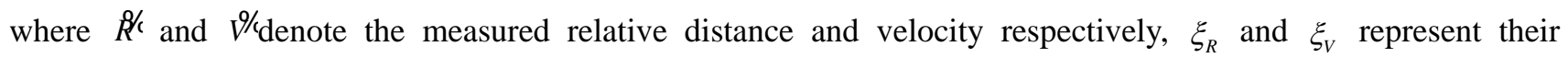
radio measurement noises respectively (assumed to be white Gaussian noise).

\section{Radio/FADS/IMU Integrated Navigation Algorithm}

In order to estimate the position and velocity of entry vehicle and suppress the measurement noises, the acceleration and angular velocity data from IMU, dynamic pressure and heating data from FADS, and range and velocity data from radio measurement are incorporated into the integrated navigation algorithm by unscented Kalman filter (UKF).

\subsection{System State Equations}

Define state variables as $\boldsymbol{x}=\left[\boldsymbol{r}^{\mathrm{T}}, \boldsymbol{v}^{\mathrm{T}}, \boldsymbol{\Omega}^{\mathrm{T}}\right]_{9}^{\mathrm{T}}$. The position vector $\boldsymbol{r}$, velocity vector $\boldsymbol{v}$, and attitude angle vector $\boldsymbol{\Omega}$ are all defined in the Mars centered inertial coordinate system. Then, the 6-DOF Mars entry dynamic equations (Eq. (4)) can be rewritten as follows:

$$
\boldsymbol{k}=\boldsymbol{f}(\boldsymbol{x})=\left[\begin{array}{c}
\boldsymbol{v} \\
\boldsymbol{T}_{V}^{I} \boldsymbol{a}_{V}+\boldsymbol{T}_{G}^{I} \boldsymbol{g}_{G} \\
\boldsymbol{K} \boldsymbol{\omega}_{B}
\end{array}\right]_{9 \times 1}
$$

\subsection{Navigation Measurement Equations}

According to the IMU measurement model (Eq. (11)), FADS measurement model (Eq. (17)) and radio measurement model (Eq. (22)), the navigation measurement equations can be rewritten as follows: 


$$
\boldsymbol{y}=\left[\begin{array}{l}
\boldsymbol{y}_{I M U} \\
\boldsymbol{y}_{F A D S} \\
\boldsymbol{y}_{\text {Radio }}
\end{array}\right]_{13 \times 1}=\boldsymbol{h}(\boldsymbol{x})+\boldsymbol{v}
$$

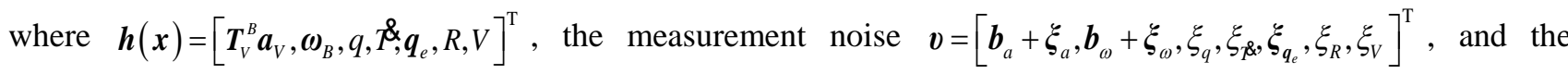
covariance matrix of measurement errors is denoted by $\boldsymbol{P}_{v}$.

\subsection{Navigation Filter}

As the dynamic equation of Mars entry is highly nonlinear system, navigation filter is designed using UKF to suppress system noise and navigation measurement noise and better estimate the state variables of the entry vehicle. The basic framework for the UKF involves estimation of the state of a discrete-time nonlinear dynamic system [12, 14]:

$$
\begin{gathered}
\boldsymbol{k}_{k+1}=\boldsymbol{f}\left(\boldsymbol{x}_{k}, \boldsymbol{u}_{k}, \boldsymbol{w}_{k}\right) \\
\boldsymbol{y}_{k}=\boldsymbol{h}\left(\boldsymbol{x}_{k}, \boldsymbol{v}_{k}\right)
\end{gathered}
$$

where $\boldsymbol{x}_{k}$ denotes the system state, $\boldsymbol{y}_{k}$ denotes the system measurement, $\boldsymbol{u}_{k}$ is the control variable. The system process noise $\boldsymbol{w}_{k}$ and observation noise $\boldsymbol{v}_{k}$ are assumed to be the zero mean white Gaussian noise and be independent of each other [12, 14-16]:

$$
E\left(\boldsymbol{w}_{k} \boldsymbol{w}_{j}^{\mathrm{T}}\right)=\boldsymbol{P}_{w} \delta_{k j}, E\left(\boldsymbol{v}_{k} \boldsymbol{v}_{j}^{\mathrm{T}}\right)=\boldsymbol{P}_{v} \delta_{k j}, E\left(\boldsymbol{w}_{k} \boldsymbol{v}_{j}^{\mathrm{T}}\right)=0
$$

Initialize:

$$
\begin{gathered}
\hat{\boldsymbol{x}}_{0}=E\left[\boldsymbol{x}_{0}\right] \\
\boldsymbol{P}_{0}=E\left[\left(\boldsymbol{x}_{0}-\hat{\boldsymbol{x}}_{0}\right)\left(\boldsymbol{x}_{0}-\hat{\boldsymbol{x}}_{0}\right)^{\mathrm{T}}\right] \\
\hat{\boldsymbol{x}}_{0}^{a}=E\left[\boldsymbol{x}_{0}^{a}\right]=\left[\begin{array}{lll}
\hat{\boldsymbol{x}}_{0}^{\mathrm{T}} & 0 & 0
\end{array}\right]^{\mathrm{T}} \\
\boldsymbol{P}_{0}^{a}=E\left[\left(\boldsymbol{x}_{0}^{a}-\hat{\boldsymbol{x}}_{0}^{a}\right)\left(\boldsymbol{x}_{0}^{a}-\hat{\boldsymbol{x}}_{0}^{a}\right)^{\mathrm{T}}\right]=\operatorname{diag}\left(\boldsymbol{P}_{0}, \boldsymbol{P}_{w}, \boldsymbol{P}_{v}\right)
\end{gathered}
$$

where $\boldsymbol{x}^{a}=\left[\begin{array}{lll}\boldsymbol{x}^{\mathrm{T}} & \boldsymbol{w}^{\mathrm{T}} & \boldsymbol{v}^{\mathrm{T}}\end{array}\right]^{\mathrm{T}}, \operatorname{diag}(\bullet)$ represents diagonal matrix.

Calculate sigma points:

$$
\chi_{k-1}^{a}=\left[\begin{array}{lll}
\hat{\boldsymbol{x}}_{k-1}^{a} & \hat{\boldsymbol{x}}_{k-1}^{a}+\sqrt{(n+\varsigma) \boldsymbol{P}_{k-1}^{a}} & \hat{\boldsymbol{x}}_{k-1}^{a}-\sqrt{(n+\varsigma) \boldsymbol{P}_{k-1}^{a}}
\end{array}\right]
$$

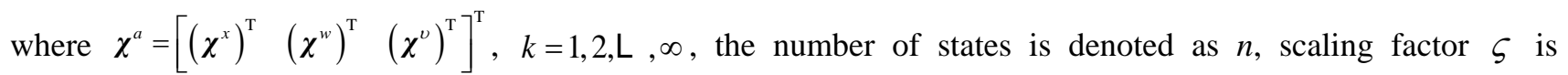
calculated as

$$
\varsigma=a^{2}(n+\kappa)-n
$$


where $a, \kappa$ are scaling parameters. $a$ is usually set to a small positive value between 0.0001 and $1, \kappa$ is often set as $0[14]$.

UKF time update:

$$
\begin{aligned}
& \mathcal{K}_{k / k-1}^{x}=\boldsymbol{f}\left(\chi_{k-1}^{x}, \chi_{k-1}^{w}\right) \\
& \hat{\boldsymbol{x}}_{k / k-1}=\sum_{i=0}^{2 n} W_{i}^{(m)} \boldsymbol{\chi}_{i, k / k-1}^{x} \\
& \boldsymbol{P}_{k / k-1}=\sum_{i=0}^{2 n} W_{i}^{(c)}\left[\chi_{i, k / k-1}^{x}-\hat{\boldsymbol{x}}_{k / k-1}\right]\left[\chi_{i, k / k-1}^{x}-\hat{\boldsymbol{x}}_{k / k-1}\right]^{\mathrm{T}} \\
& \boldsymbol{\gamma}_{k / k-1}=\boldsymbol{h}\left(\boldsymbol{\chi}_{k-1}^{x}, \boldsymbol{\chi}_{k-1}^{v}\right) \\
& \hat{\boldsymbol{y}}_{k / k-1}=\sum_{i=0}^{2 n} W_{i}^{(m)} \boldsymbol{\gamma}_{i, k / k-1}^{x}
\end{aligned}
$$

where the weight coefficients $W_{i}^{(m)}$ and $W_{i}^{(c)}$ are defined as follows:

$$
\begin{gathered}
W_{0}^{(m)}=\frac{\varsigma}{n+\varsigma} \\
W_{0}^{(c)}=\frac{\varsigma}{n+\varsigma}+\left(1-a^{2}+b\right) \\
W_{i}^{(m)}=W_{i}^{(c)}=\frac{1}{2(n+\varsigma)}, i=1, \mathrm{~L}, 2 n
\end{gathered}
$$

where the scaling parameter $b$ is usually set as 2 for Gaussian distributions [14].

UKF measurement update:

$$
\begin{aligned}
& \boldsymbol{P}_{y_{R} \varphi_{k}}=\sum_{i=0}^{2 n} W_{i}^{(c)}\left[\boldsymbol{\gamma}_{i, k / k-1}-\hat{\boldsymbol{y}}_{k / k-1}\right]\left[\boldsymbol{\gamma}_{i, k / k-1}-\hat{\boldsymbol{y}}_{k / k-1}\right]^{\mathrm{T}} \\
& \boldsymbol{P}_{x_{k} y_{k}}=\sum_{i=0}^{2 n} W_{i}^{(c)}\left[\boldsymbol{\chi}_{i, k / k-1}-\hat{\boldsymbol{x}}_{k / k-1}\right]\left[\boldsymbol{\gamma}_{i, k / k-1}-\hat{\boldsymbol{y}}_{k / k-1}\right]^{\mathrm{T}}
\end{aligned}
$$

UKF filter update:

$$
\begin{aligned}
& \boldsymbol{K}_{k}=\boldsymbol{P}_{x_{k} y_{k}} \boldsymbol{P}_{y_{k} g_{k}}^{-1} \\
& \hat{\boldsymbol{x}}_{k}=\hat{\boldsymbol{x}}_{k / k-1}+\boldsymbol{K}_{k}\left(\boldsymbol{y}_{k}-\hat{\boldsymbol{y}}_{k / k-1}\right) \\
& \boldsymbol{P}_{k}=\boldsymbol{P}_{k / k-1}-\boldsymbol{K}_{k} \boldsymbol{P}_{9 \rho_{0} 9 \rho_{R}} \boldsymbol{K}_{k}^{\mathrm{T}}
\end{aligned}
$$

To avoid the possible singularity occurs during inversion calculation, the gain matrix $\boldsymbol{K}_{k}$ in Eq. (44) can be obtained using UD decomposition [14]. As $\boldsymbol{P}_{\vartheta_{\mathrm{R}} \mathrm{R}_{\mathrm{R}}}$ is a symmetric positive definite matrix, we can assumed that

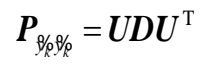

where $\boldsymbol{U}$ is an unit upper triangular matrix, $\boldsymbol{D}$ is a diagonal matrix. 
Then, Eq. (44) and Eq. (43) can be respectively substituted as follows

$$
\begin{gathered}
\boldsymbol{K}_{k}=\boldsymbol{P}_{x_{k} y_{k}}\left(\boldsymbol{U} \boldsymbol{D} \boldsymbol{U}^{\mathrm{T}}\right)^{-1} \\
\boldsymbol{P}_{x_{k} y_{k}}=\boldsymbol{K}_{k} \boldsymbol{U} \boldsymbol{D} \boldsymbol{U}^{\mathrm{T}}
\end{gathered}
$$

\subsection{Observability Analysis}

Because the observability and observable degree directly determine the performance of the navigation algorithm, observability analysis is an important work for the integrated navigation. The observability of both IMU/surface radio beacons and IMU/orbiting radio beacons integrated navigation had been discussed in the previous work [18-22]. The aim of this paper is to develop a new integrated navigation algorithm for Mars atmospheric entry. Therefore, the observability analysis of IMU/FADS/radio integrated navigation is conducted using the principle of observability rank analysis.

According to the linear system theory, the nonlinear dynamic equations (Eq. (23)) and measurement equations (Eq. (24)) are linearized along the reference trajectory. The reference trajectory is obtained from the entry dynamics driven by the MSL guidance [32]. Define the Jacobian matrix of partial derivatives of $\boldsymbol{f}$ with respect to state variable $\boldsymbol{x}$, that is:

$$
\boldsymbol{F}_{k / k-1}=\left.\frac{\partial \boldsymbol{f}(\boldsymbol{x}, k)}{\partial \boldsymbol{x}}\right|_{x=x_{k-1}}=\left[\begin{array}{ccc}
\boldsymbol{0}_{3 \times 3} & \boldsymbol{I}_{3 \times 3} & \boldsymbol{0}_{3 \times 3} \\
\frac{\partial\left(\boldsymbol{T}_{V}^{I} \boldsymbol{a}_{V}+\boldsymbol{T}_{G}^{I} \boldsymbol{g}_{G}\right)}{\partial \boldsymbol{r}} & \boldsymbol{0}_{3 \times 3} & \frac{\partial\left(\boldsymbol{T}_{V}^{I} \boldsymbol{a}_{V}+\boldsymbol{T}_{G}^{I} \boldsymbol{g}_{G}\right)}{\partial \boldsymbol{\Omega}} \\
\boldsymbol{0}_{3 \times 3} & \boldsymbol{0}_{3 \times 3} & \frac{\partial\left(\boldsymbol{K} \boldsymbol{\omega}_{B}\right)}{\partial \boldsymbol{\Omega}}
\end{array}\right]_{9 \times 9}
$$

Define the sensitivity matrix:

$$
\boldsymbol{H}_{k}=\left.\frac{\partial \boldsymbol{h}(\boldsymbol{x})}{\partial \boldsymbol{x}}\right|_{x=\hat{\boldsymbol{x}}_{k}}=\left[\begin{array}{ccc}
\boldsymbol{0}_{3 \times 3} & \boldsymbol{0}_{3 \times 3} & \frac{\partial\left(\boldsymbol{T}_{V}^{B} \boldsymbol{a}_{V}\right)}{\partial \boldsymbol{\Omega}^{\mathrm{T}}} \\
\boldsymbol{0}_{3 \times 3} & \boldsymbol{0}_{3 \times 3} & \frac{\partial \boldsymbol{\omega}_{B}}{\partial \boldsymbol{\Omega}^{\mathrm{T}}} \\
\boldsymbol{0}_{1 \times 3} & \frac{\partial q}{\partial \boldsymbol{v}^{\mathrm{T}}} & \boldsymbol{0}_{1 \times 3} \\
\boldsymbol{0}_{1 \times 3} & \frac{\partial T^{\mathcal{E}}}{\partial \boldsymbol{v}^{\mathrm{T}}} & \boldsymbol{0}_{1 \times 3} \\
\boldsymbol{0}_{3 \times 3} & \frac{\partial \boldsymbol{q}_{e}}{\partial \boldsymbol{v}^{\mathrm{T}}} & \frac{\partial \boldsymbol{q}_{e}}{\partial \boldsymbol{\Omega}^{\mathrm{T}}} \\
\frac{\partial R}{\partial \boldsymbol{r}^{\mathrm{T}}} & \boldsymbol{0}_{1 \times 3} & \boldsymbol{0}_{1 \times 3} \\
\frac{\partial V}{\partial \boldsymbol{r}^{\mathrm{T}}} & \frac{\partial V}{\partial \boldsymbol{v}^{\mathrm{T}}} & \boldsymbol{0}_{1 \times 3}
\end{array}\right]_{13 \times 9}
$$

Once a specific moment is selected, the certain $\boldsymbol{F}$ and $\boldsymbol{H}$ can be determined, and then the observability 
matrix can be constructed. The nonlinear system of integrated navigation is transformed into a linear system (state equation and output equation) as follows

$$
\begin{cases}\boldsymbol{\&}=\boldsymbol{A x}+\boldsymbol{B u} & \boldsymbol{A}=\boldsymbol{F}_{k / k-1} \\ \boldsymbol{y}=\boldsymbol{C} \boldsymbol{x}+\boldsymbol{D u} & \boldsymbol{C}=\boldsymbol{H}_{k}\end{cases}
$$

where $\boldsymbol{A}$ and $\boldsymbol{C}$ are system matrix and output matrix respectively, input vector $\boldsymbol{u}=[\sigma]$. Input matrix $\boldsymbol{B}$ and feed-forward matrix $\boldsymbol{D}$ are respectively defined as follows

$$
\begin{gathered}
\boldsymbol{B}_{k / k-1}=\left.\frac{\partial \boldsymbol{f}(\boldsymbol{x}, k)}{\partial \boldsymbol{u}}\right|_{\boldsymbol{x}=\boldsymbol{x}_{k-1}}=\left[\begin{array}{c}
\boldsymbol{0}_{3 \times 1} \\
\frac{\partial\left(\boldsymbol{T}_{V}^{I} \boldsymbol{a}_{V}+\boldsymbol{T}_{G}^{I} \boldsymbol{g}_{G}\right)}{\partial \boldsymbol{u}} \\
\frac{\partial\left(\boldsymbol{K} \boldsymbol{\omega}_{B}\right)}{\partial \boldsymbol{u}}
\end{array}\right]_{9 \times 1}^{\mathrm{T}} \\
\boldsymbol{D}_{k}=\left.\frac{\partial \boldsymbol{h}(\boldsymbol{x})}{\partial \boldsymbol{u}}\right|_{\boldsymbol{x}=\hat{\boldsymbol{x}}_{k}}=\left[\frac{\partial\left(\boldsymbol{T}_{V}^{B} \boldsymbol{a}_{V}\right)}{\partial \boldsymbol{u}}, \frac{\partial \boldsymbol{\omega}_{B}}{\partial \boldsymbol{u}}, 0,0, \frac{\partial \boldsymbol{q}_{e}}{\partial \boldsymbol{u}}, 0,0\right]_{13 \times 1}^{\mathrm{T}}
\end{gathered}
$$

Then, the observability matrix $\boldsymbol{G}$ is defined as

$$
\boldsymbol{G}=\left[\begin{array}{c}
\boldsymbol{C} \\
\boldsymbol{C A} \\
\mathrm{M} \\
\boldsymbol{C A}^{8}
\end{array}\right]
$$

With this reduced linear system description, the observability matrix has also rank of 9 at any linearization point along the reference trajectory. Obviously, the rank of the observability matrix is full because the dimension of system state is 9. Therefore, we can safely arrive at the conclusion that all the state variables are observable in this Radio/FADS/IMU integrated navigation system, and the navigation estimation errors should be well convergent in theory. Essentially, all the system states $\left(\boldsymbol{x}=\left[\boldsymbol{r}^{\mathrm{T}}, \boldsymbol{v}^{\mathrm{T}}, \boldsymbol{\Omega}^{\mathrm{T}}\right]_{9}^{\mathrm{T}}\right)$ can be observed through directly integrating the outputs of IMU. The radio measurement is utilized to partly suppress the system/measurement noise and improve the estimation accuracy of position and velocity. In addition, the FADS measurement is also introduced to further suppress the system/measurement noise and enhance the estimation accuracy of tri-axial velocity and tri-axial attitude angles.

\section{Simulation and Results}

In order to confirm the validity of Mars entry integrated navigation schemes presented in this paper, computer simulation in MATLAB has been carried out. MSL guidance [32] provides commanded bank angle and bank angular rate to drive the simulation procedures. Because the MSL entry phase lasts about 4 minutes, the simulated 
Mars entry span is set to be 250s and simulation step is set to be one second. We assumed that only one orbiting radio beacon is available during Mars entry. The physical parameters of Mars and the entry vehicle are given in Table I. The initial state variables and initial errors of the entry vehicle are listed in Table II. An optimal orbit is selected according to the previous works [21, 22], and the initial state variables and initial errors of the orbiter are listed in Table III. Four-order Runge-Kutta algorithm is selected as numerical solver of integral the Mars entry dynamic equations in Eqs. (1) and (4).

TABLE I

PHYSICAL PARAMETERS OF MARS AND ENTRY VEHICLE

\begin{tabular}{lcc}
\hline \hline Parameters & value & unit \\
\hline Mars reference radius, $r_{0}$ & $3397 \times 10^{3}$ & $\mathrm{~m}$ \\
Mars gravitational constant, $\mu$ & $4.2828 \times 10^{13}$ & $\mathrm{~m}^{3} / \mathrm{s}^{2}$ \\
Mars rotation angular rate, $\omega_{M}$ & $7.0882 \times 10^{-5}$ & $\mathrm{rad} / \mathrm{s}$ \\
Entry vehicle ballistic coefficient, $m / C_{D} S$ & 117 & $\mathrm{~kg} / \mathrm{m}^{2}$ \\
Lift-drag ratio, $L / D$ & 0.24 & - \\
Vehicle nose radius, $r_{n}$ & 0.66 & $\mathrm{~m}$ \\
\hline \hline
\end{tabular}

TABLE II

INITIAL STATE AND INITIAL ERRORS OF ENTRY VEHICLE

\begin{tabular}{cccc}
\hline \hline Entry state & Initial values & Initial errors & unit \\
\hline $\boldsymbol{r}$ & {$\left[3522 \times 10^{3}, 2000,-2000\right]$} & {$[1000,-943,1057]$} & $\mathrm{m}$ \\
$\boldsymbol{v}$ & {$[-1256,2075,5496]$} & {$[5,-5,5]$} & $\mathrm{m} / \mathrm{s}$ \\
$\boldsymbol{\Omega}$ & {$[0,-0.47,1.21]$} & {$\left[10^{-2}, 10^{-2}, 10^{-2}\right]$} & $\mathrm{rad}$ \\
\hline \hline
\end{tabular}

TABLE III

INITIAL STATE AND INITIAL ERRORS OF ORBITER

\begin{tabular}{cccc}
\hline \hline Orbiter states & Initial values & Initial errors & unit \\
\hline $\boldsymbol{r}_{o}$ & {$[7855.7,-461.8,749.8] \times 10^{3}$} & {$[0.1,0.1,0.1] \times 10^{3}$} & $\mathrm{~m}$ \\
$\boldsymbol{v}_{o}$ & {$[66.2,2206.4,-413]$} & {$[0.1,0.1,0.1]$} & $\mathrm{m} / \mathrm{s}$ \\
\hline \hline
\end{tabular}

Based on the detailed parameters of the LN-200 IMU [12, 13], the accelerometer bias $\boldsymbol{b}_{a}$ and gyro bias $\boldsymbol{b}_{\omega}$ 
used in our simulation are assumed to be $\left[3 \times 10^{-3}, 3 \times 10^{-3}, 3 \times 10^{-3}\right] \mathrm{m} / \mathrm{s}^{2}$ and $\left[5 \times 10^{-6}, 5 \times 10^{-6}, 5 \times 10^{-6}\right] \mathrm{rad} / \mathrm{s}$ respectively, the covariance of white Gaussian noise $\boldsymbol{\xi}_{a}$ and $\boldsymbol{\xi}_{\omega}$ are set to be $\left[5 \times 10^{-8}, 5 \times 10^{-8}, 5 \times 10^{-8}\right]\left(\mathrm{m} / \mathrm{s}^{2}\right)^{2}$ and $\left[9 \times 10^{-8}, 9 \times 10^{-8}, 9 \times 10^{-8}\right](\mathrm{rad} / \mathrm{s})^{2}$ respectively. The covariance of Radio range and velocity measurement noise $\xi_{R}$ and $\xi_{V}$ in the navigation measurement model are set as $400 \mathrm{~m}^{2}$ and $0.4(\mathrm{~m} / \mathrm{s})^{2}$ respectively. According to the desired index data of the FADS developing for China planetary reentry/entry vehicles [23-25, 29], the covariance of white Gaussian noises $\xi_{q}, \xi_{\mathbb{R}}, \xi_{q_{e}}$ are set as $4 \times 10^{2} \mathrm{~Pa}^{2}, 16 \times 10^{-2}(\mathrm{~K} / \mathrm{s})^{2}$, and $\left[4 \times 10^{-4}, 4 \times 10^{-4}, 4 \times 10^{-4} \mathrm{rad}^{2}\right.$ respectively. The integrated navigation filter parameters are defined in Table IV.

\section{TABLE IV}

INTEGRATED NAVIGATION FILTER PARAMETERS

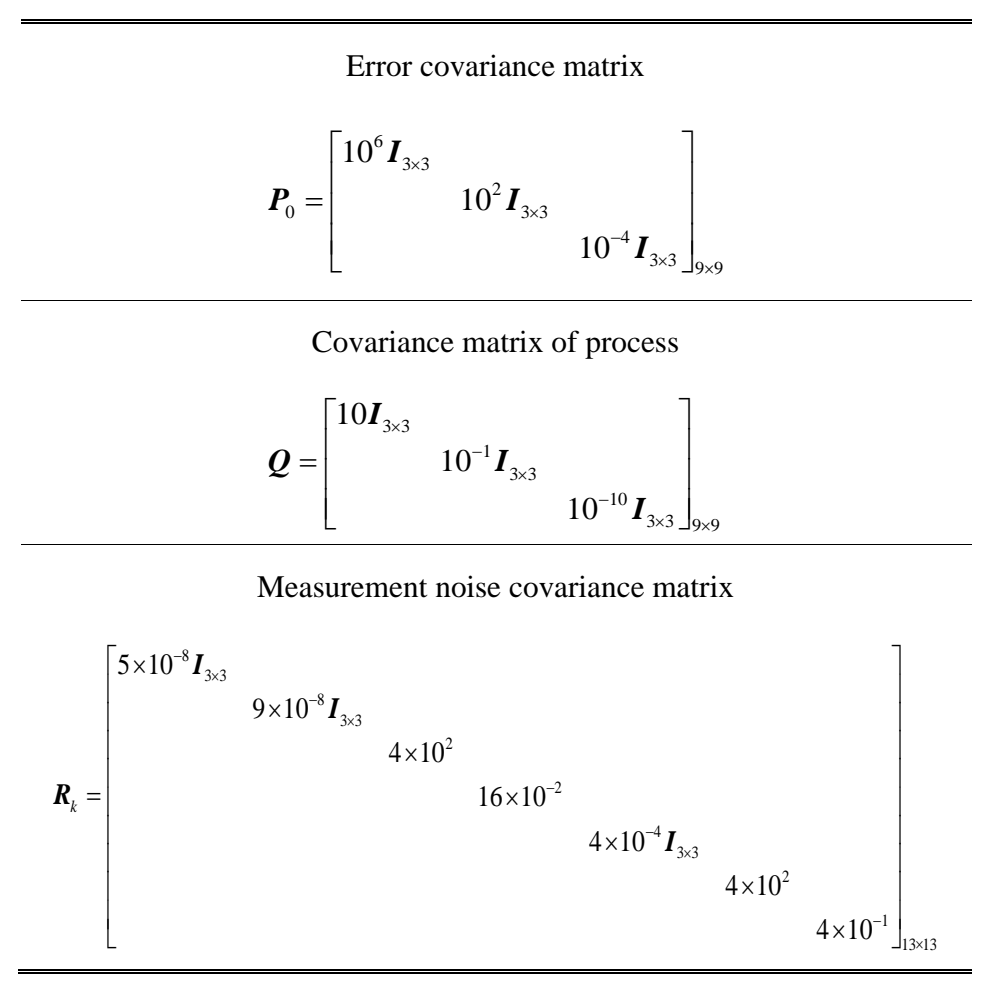

As mentioned in the Section 1, for Mars orbiting/landing collaborative exploration mission, the performances of four Mars entry navigation solutions are tested for comparison in the following simulations. They are traditional inertial navigation, radio/IMU integrated navigation, FADS/IMU integrated navigation, and radio/FADS/IMU integrated navigation.

The results obtained in the simulations are the states' estimation errors, i.e. actual state minus estimated state. And the actual state is generated from the entry dynamics that driven by the MSL guidance. The estimated state is obtained by the navigation filter fusing the measurements from radio, FADS, and IMU.

\subsection{Traditional inertial navigation}


Tri-axial position estimation errors, velocity estimation errors and attitude estimation errors from traditional IMU based dead reckoning navigation are shown in Fig.2 to Fig.4 respectively, and corresponding total position estimation errors and velocity estimation errors from traditional IMU based dead reckoning navigation are shown in Figs. 5 and 6 respectively.

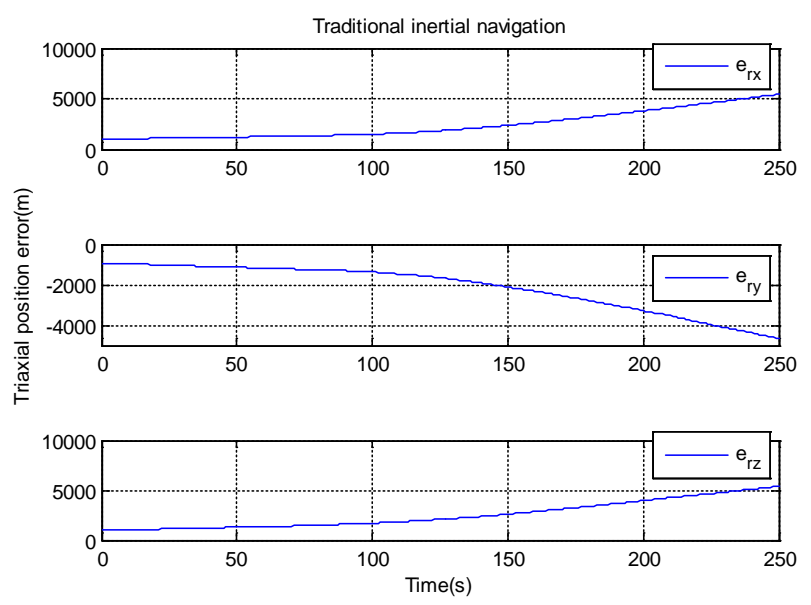

Fig. 2. Tri-axial position errors from traditional inertial navigation.

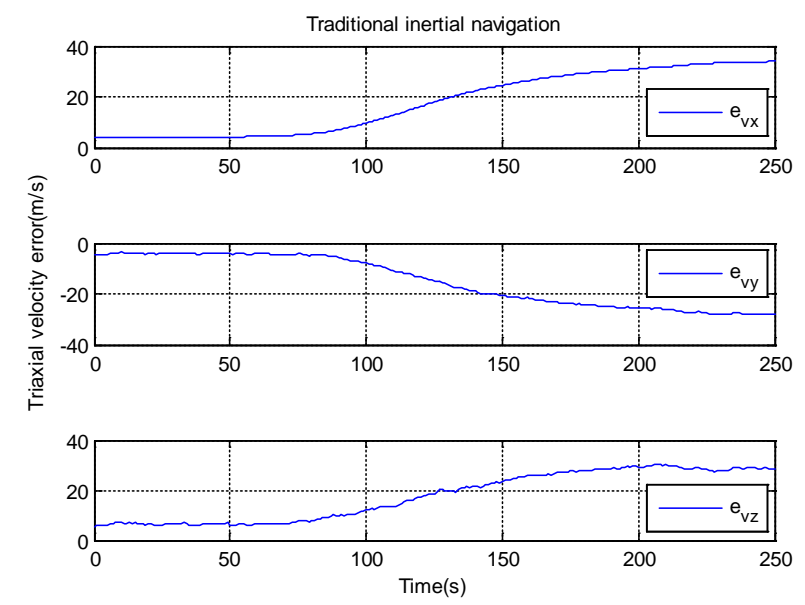

Fig. 3. Tri-axial velocity errors from traditional inertial navigation. 

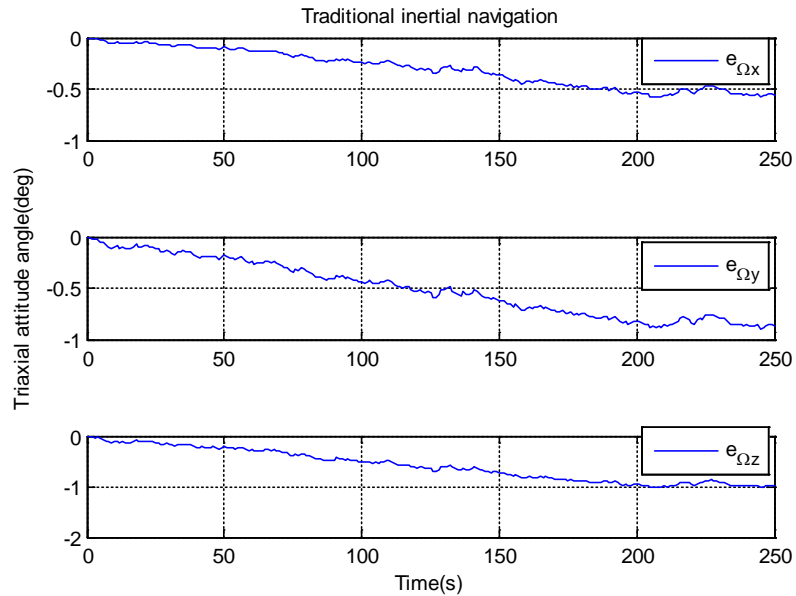

Fig. 4. Tri-axial attitude errors from traditional inertial navigation.

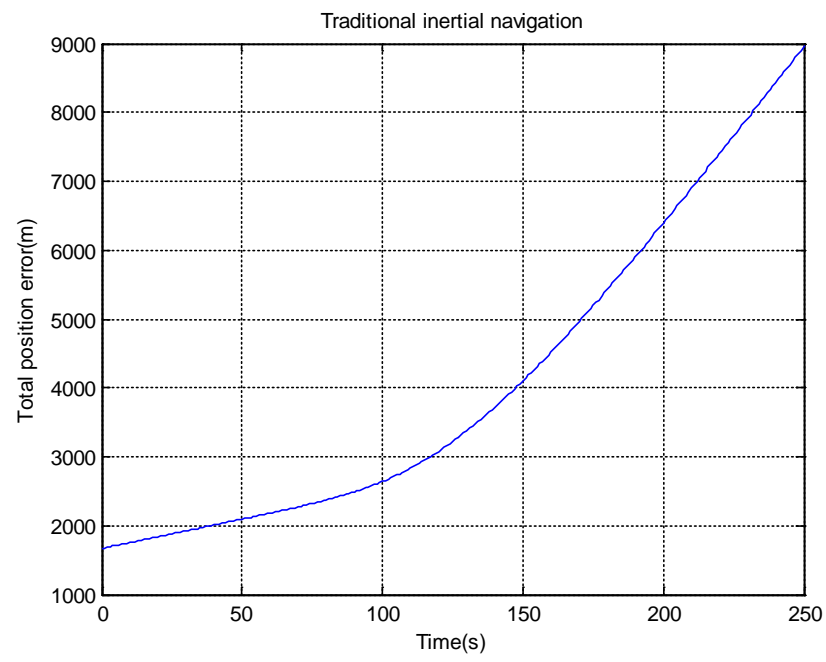

Fig. 5. Total position error from traditional inertial navigation.

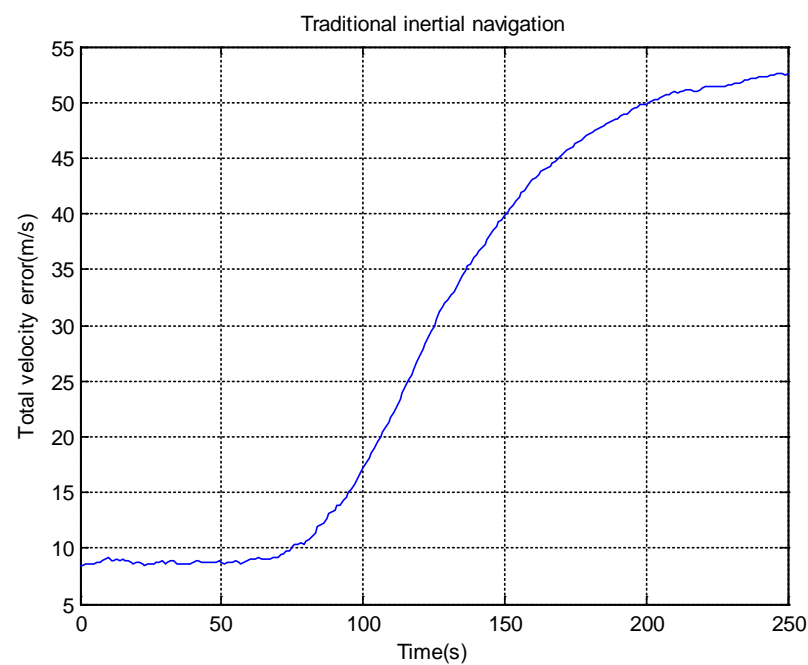

Fig. 6. Total velocity error from traditional inertial navigation. 
It can be seen from Figs. 2 and 3 that tri-axial position and velocity errors are about $6 \mathrm{~km}$ and $30 \mathrm{~m} / \mathrm{s}$ respectively due to the inertial drift and bias. Moreover, it is easily concluded from the above simulation results shown in Figs. 5 and 6 that the estimation errors from IMU based dead reckoning navigation gradually diverge, and the final position and velocity errors reach about $9 \mathrm{~km}$ and $50 \mathrm{~m} / \mathrm{s}$ under the current simulation assumptions. Such large navigation errors cannot meet the requirements of future Mars orbiting and landing collaborative missions.

\subsection{Radio/IMU integrated navigation with one orbiting radio beacon}

For Chinese Mars orbiting/landing collaborative mission, only one orbiter is available to support the entry navigation after the separation between the orbiter and the entry vehicle. Therefore, only one orbiting radio beacon is considered in the following simulation case. The relative geometrical relationship between the orbiter and the entry vehicle is shown in Fig.7. Tri-axial position estimation errors, velocity estimation errors and attitude estimation errors are shown in Fig.8 to Fig.10 respectively, and corresponding total position estimation errors and velocity estimation errors are shown in Figs. 11 and 12 respectively.

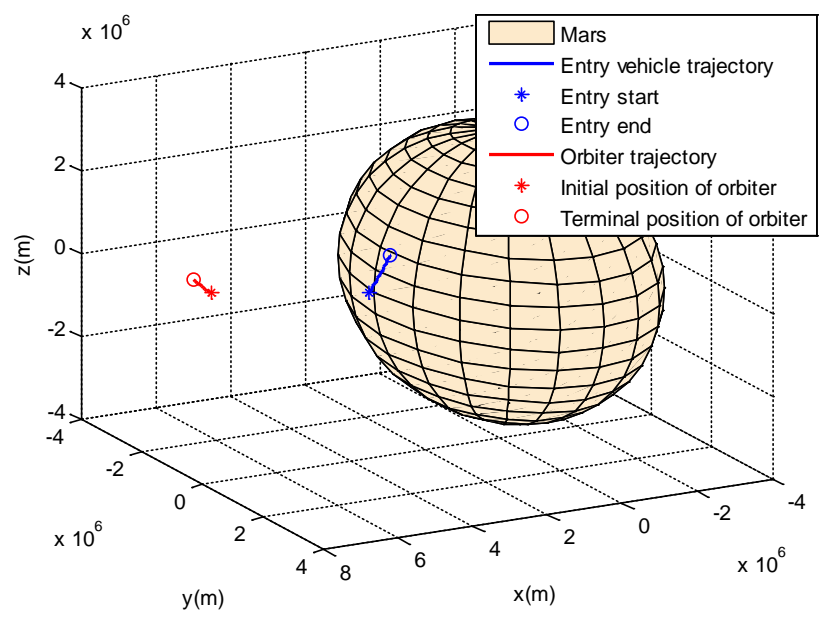

Fig. 7. Relative geometrical relationship between the orbiter and the entry vehicle. 

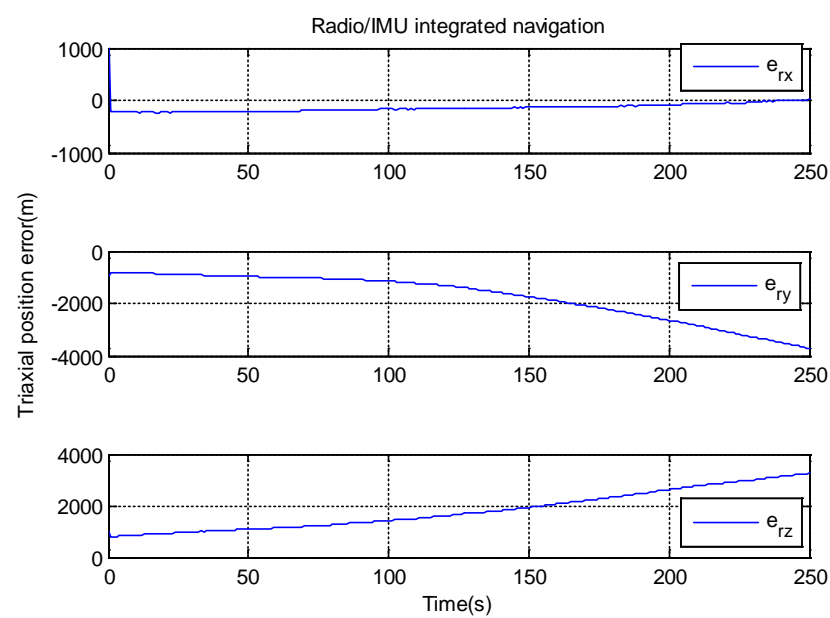

Fig. 8. Tri-axial position errors from radio/IMU integrated navigation with one orbiting beacon.
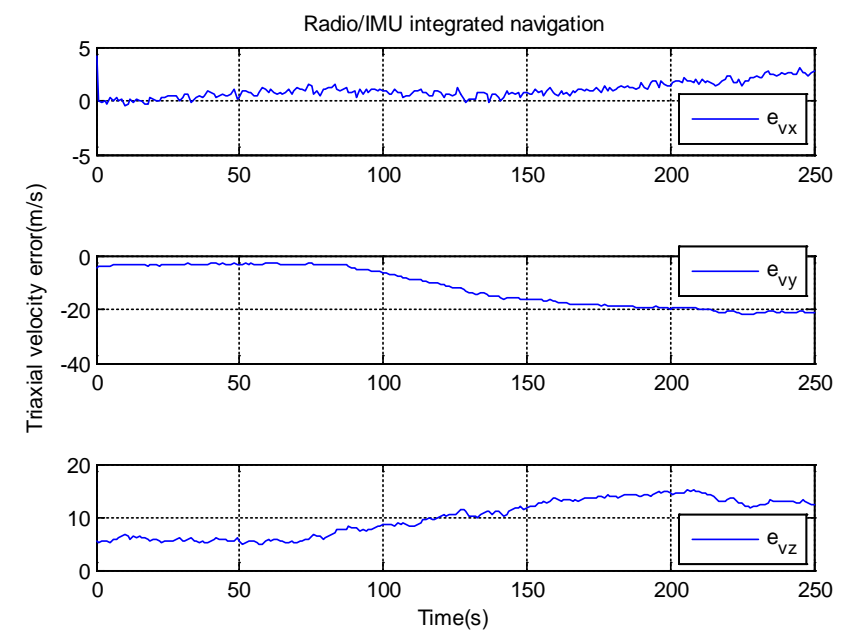

Fig.9. Tri-axial velocity errors from radio/IMU integrated navigation with one orbiting beacon.

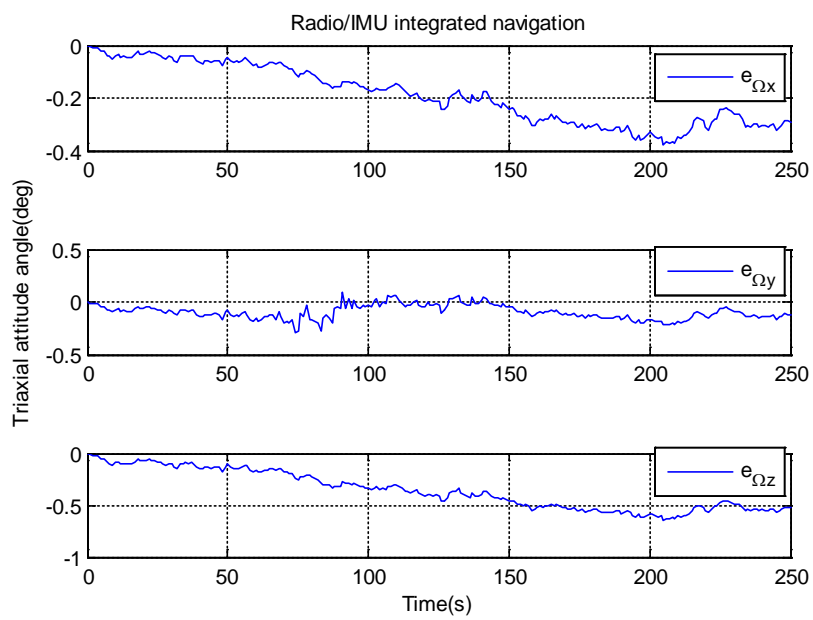

Fig.10. Tri-axial attitude errors from radio/IMU integrated navigation with one orbiting beacon. 


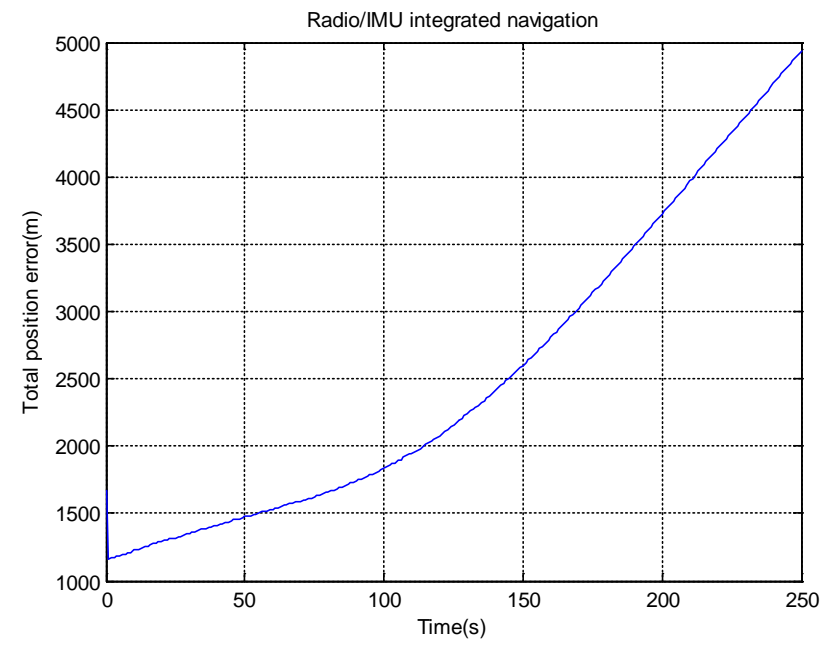

Fig.11. Total position error from radio/IMU integrated navigation with one orbiting beacon.

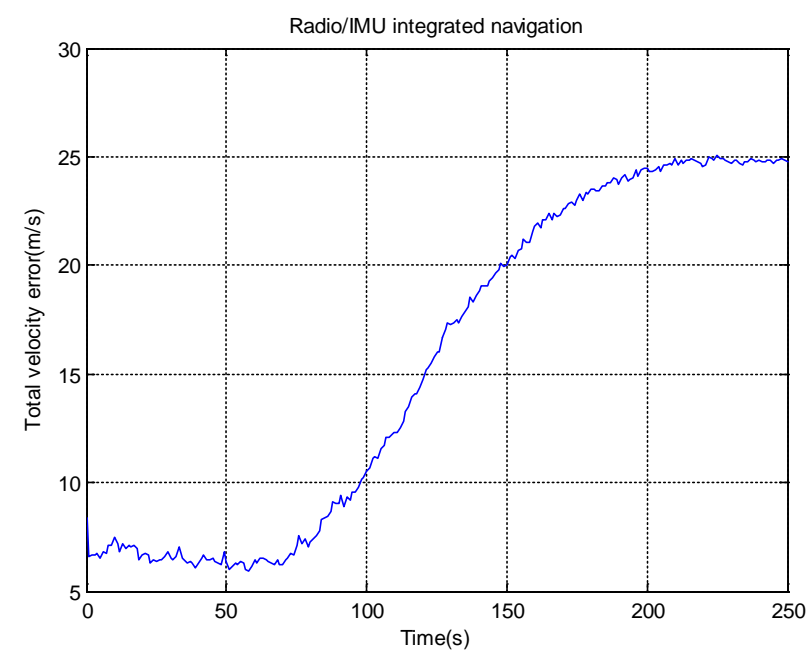

Fig.12. Total velocity error from radio/IMU integrated navigation with one orbiting beacon.

According to the simulation results shown in Figs. 8 to 12, it is concluded that the major trends of the navigation errors are also gradually divergent, and the navigation accuracy is slightly better than that of the traditional inertial navigation. The final position and velocity errors reach about $5 \mathrm{~km}$ and $25 \mathrm{~m} / \mathrm{s}$ under the current simulation assumptions. At the same time, it also should be noticed that the magnitude of $x$-axial position and velocity errors is smaller than those of the other two axial errors. The reason why x-axial errors have smaller magnitude than the others may lie in the geometry relation between the orbiter and the entry vehicle has a significant effect on the navigation observability degree along three different axes [20-22]. In this simulation scenario, the attitude accuracy has also been slightly improved, because tri-axial attitude errors are influenced by tri-axial position and velocity errors and commanded bank angle (see Eq.(8)). In general, such large navigation errors are also risky for Mars 
landing and hardly meet the requirements of future Mars orbiting/landing collaborative missions.

\subsection{FADS/IMU integrated navigation}

In case the orbiting radio beacon was unavailable, the FADS/IMU integrated navigation could be the alternative. Tri-axial position estimation errors, velocity estimation errors and attitude estimation errors from FADS/IMU integrated navigation are shown in Fig.13 to Fig.15 respectively, and corresponding total position estimation errors and velocity estimation errors are shown in Figs. 16 and 17 respectively.

Based on the simulation results shown in Figs. 13 to 17, it can be concluded that the major trends of the navigation errors are partly divergent, and the navigation accuracy is greatly improved than that of the traditional inertial navigation. It can also be seen that the final position and velocity errors reach about $4.5 \mathrm{~km}$ and $15 \mathrm{~m} / \mathrm{s}$ under the current simulation assumptions, which can roughly meet the requirements of future Mars orbiting/landing collaborative missions. Because the FADS can not directly sense vehicle's position and flight altitude, the magnitude of position errors can be only indirectly suppressed through more credible velocity and attitude estimations, which leads to the major trends of the tri-axial position errors divergent as shown in Fig. 13. It can be easily noted from Fig.14 that $\mathrm{x}$-axis and z-axial velocity errors are convergent, but y-axis velocity error is slightly divergent. This is because the variation of velocity component along y-axis is so small that cannot be precisely sensed by the FADS, and then corresponding y-axis velocity errors cannot be effectively reduced. Fig.15 shows that the tri-axial attitude errors are well convergent, which is a benefit from completely attitude measurements from the FADS. The tri-axial attitude and total velocity errors respectively plotted in Fig.15 and Fig.17 unfold an interesting phenomenon that several slightly convergent trends exist in the course of divergence. This is because the bank angle is obviously changed several times during Mars entry phase with MSL guidance, the drift errors will be partly offset when the sign of the bank angle switches. Just as the relations defined in Eq.(8) and Eq.(16), tri-axial attitude angular rates and velocity of the entry vehicle are essentially closely related to the bank angle (control variable) that impacts on the system matrix, output matrix, and observability matrix (see Eqs.(52) to (55)). 

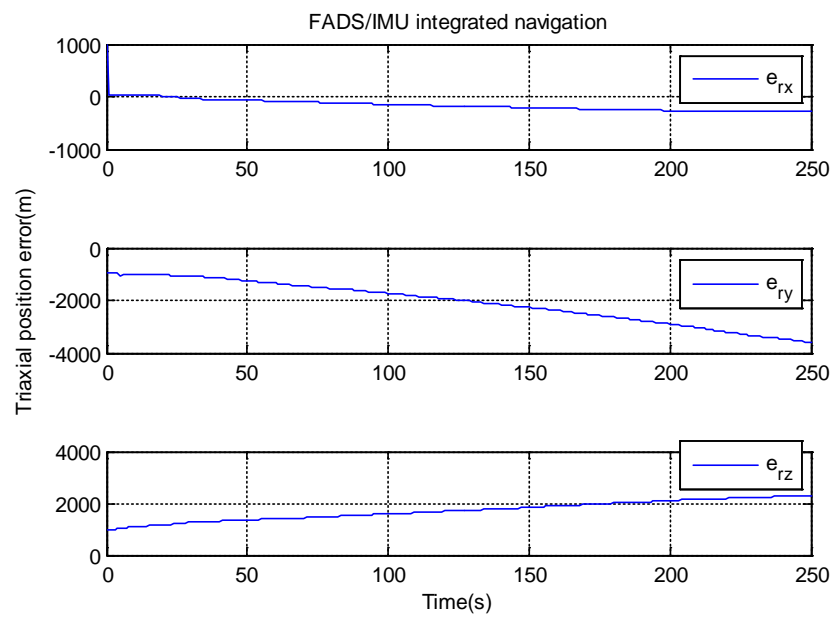

Fig.13. Tri-axial position errors from FADS/IMU integrated navigation.
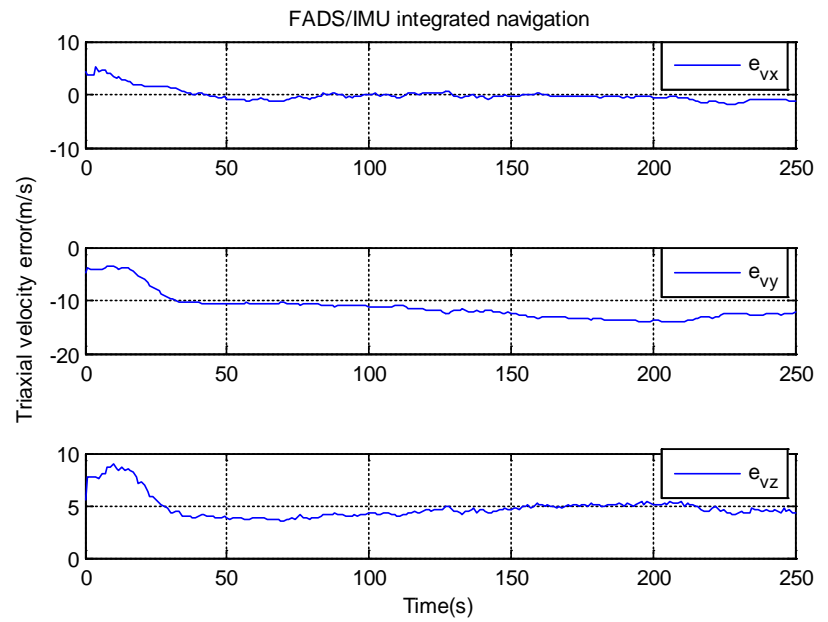

Fig.14. Tri-axial velocity errors from FADS/IMU integrated navigation.
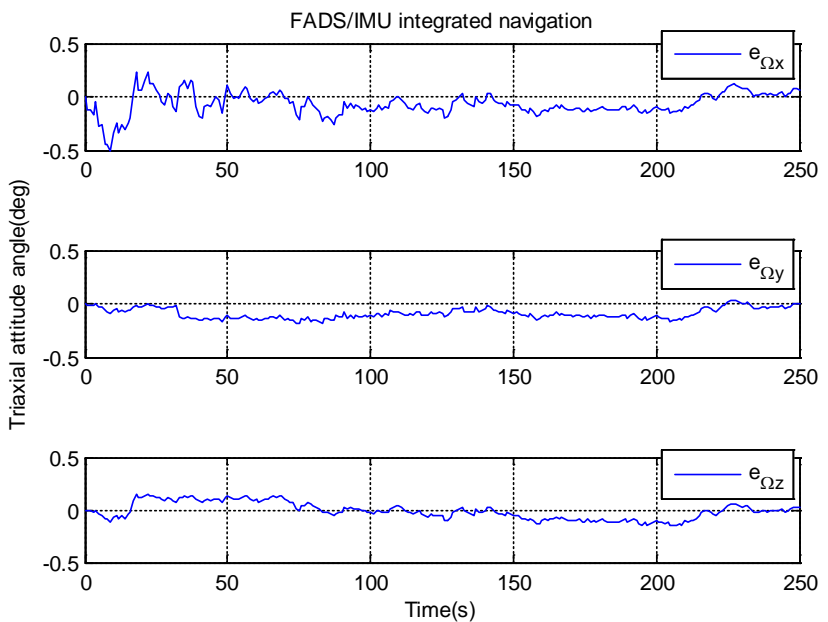

Fig.15. Tri-axial attitude errors from FADS/IMU integrated navigation. 


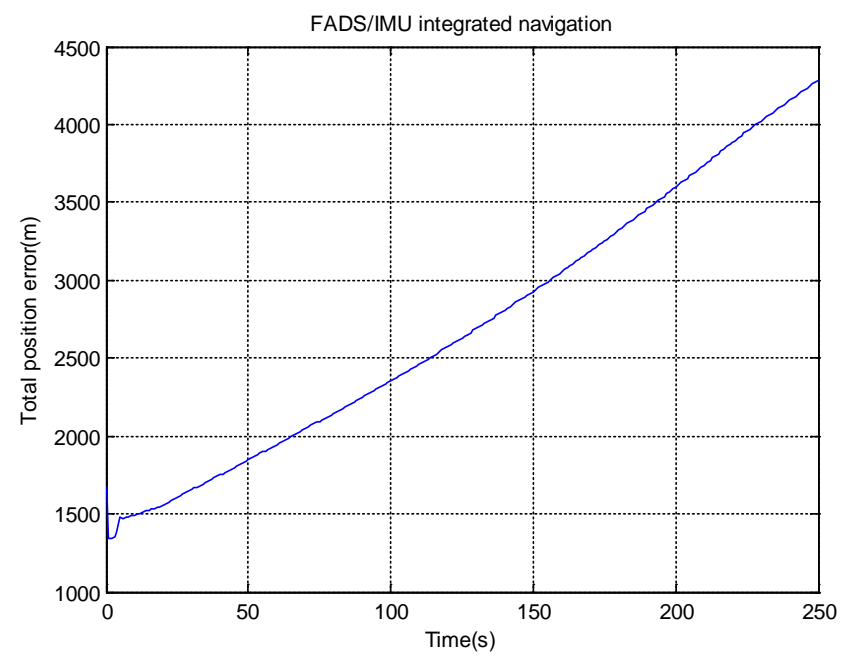

Fig.16. Total position error from FADS/IMU integrated navigation.

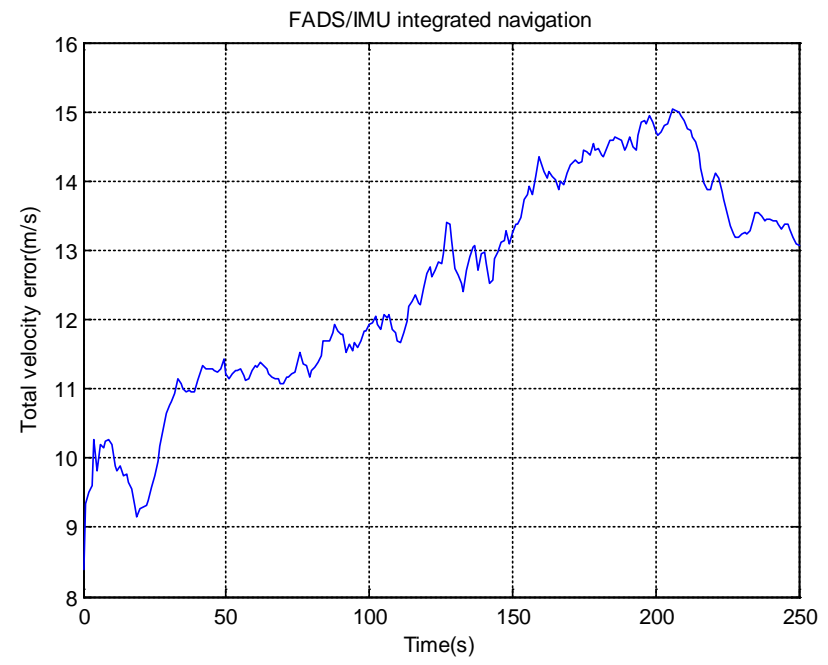

Fig.17. Total velocity error from FADS/IMU integrated navigation.

\subsection{Radio/FADS/IMU integrated navigation}

In this simulation case, one orbiting radio beacon and onboard FADS are included as the external navigation sensors. Because the radio measurements and FADS outputs are included to correct the measurement noise from IMU bias and drift, the accuracy of radio/FADS/IMU integrated navigation is likely to be greatly improved than IMU based dead reckoning navigation. Tri-axial position estimation errors, velocity estimation errors and attitude estimation errors from radio/FADS/IMU integrated navigation are plotted in Figs. 18 to 19 respectively, and corresponding total position estimation errors and velocity estimation errors from radio/FADS/IMU are also shown in Figs. 21 and 23 respectively. Besides, the radio/FADS/IMU integrated navigation can also output the estimated Mars atmospheric density during entry phase as shown in Fig.23. 
As expected, it can be seen from the simulation results depicted in Figs.18 to 22 that both the position estimation errors and velocity estimation errors of the radio/FADS/IMU integrated navigation can be clearly reduced to a smaller magnitude when compared with those of the three navigation scenarios mentioned above, which benefit from completely observability of this integrated navigation scenario. Tri-axial position and velocity errors are about $50 \mathrm{~m}$ and $2 \mathrm{~m} / \mathrm{s}$ respectively. The filter convergence of radio/FADS/IMU integrated navigation is quite good and the position errors less than $200 \mathrm{~m}$ and velocity errors less than $6 \mathrm{~m} / \mathrm{s}$, which can completely meet the navigation needs of Mars orbiting/landing collaborative mission.

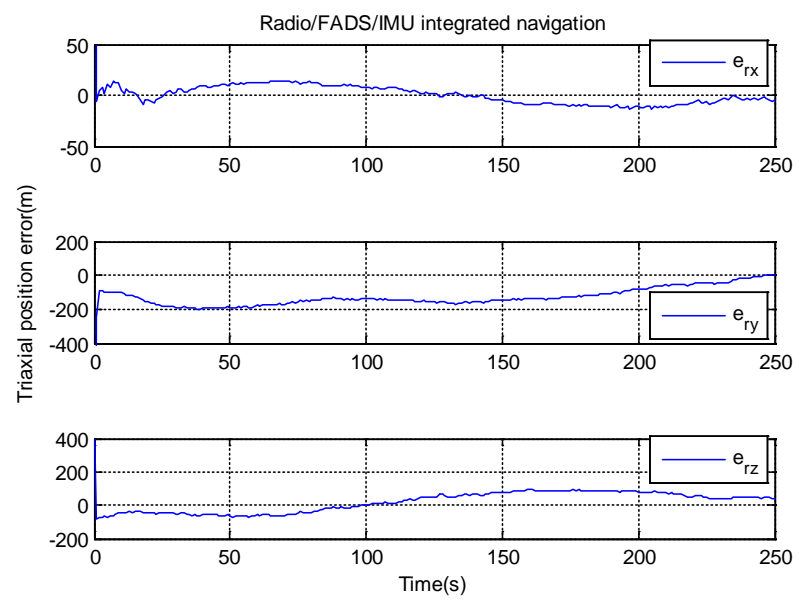

Fig.18. Tri-axial position errors from Radio/FADS/IMU integrated navigation.

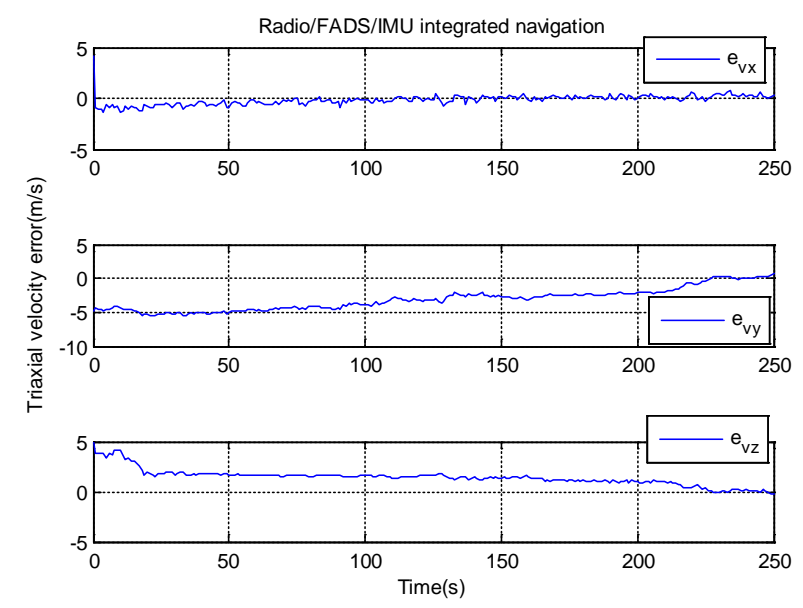

Fig.19. Tri-axial velocity errors from Radio/FADS/IMU integrated navigation.

Essentially, the main functions of radio measurements, dynamic pressure and heating measurements are respectively to determine total position and total velocity, velocity vector and attitude vector. At the same time, it should also be noticed that the magnitude of $\mathrm{x}$-axial position and velocity accuracy is almost fourfold better than 
that of the other two axial errors. The reason why x-axial errors have smaller magnitude than the others may lie in: (1) the main components of FADS measurements distribute along the x-axis while the measurements along other two orthogonal axes are smaller, and also the change rate of former is easer to be precisely sensed than that of the latter; (2) the geometry relationship of the orbiter and entry vehicle has a great influence on the degree of observability along three different axes, which mainly reflects in the total position and velocity errors. In addition, Mars atmospheric density can be accurately estimated according to Eqs.(12) to (14), which is a benefit from more precise state estimation.

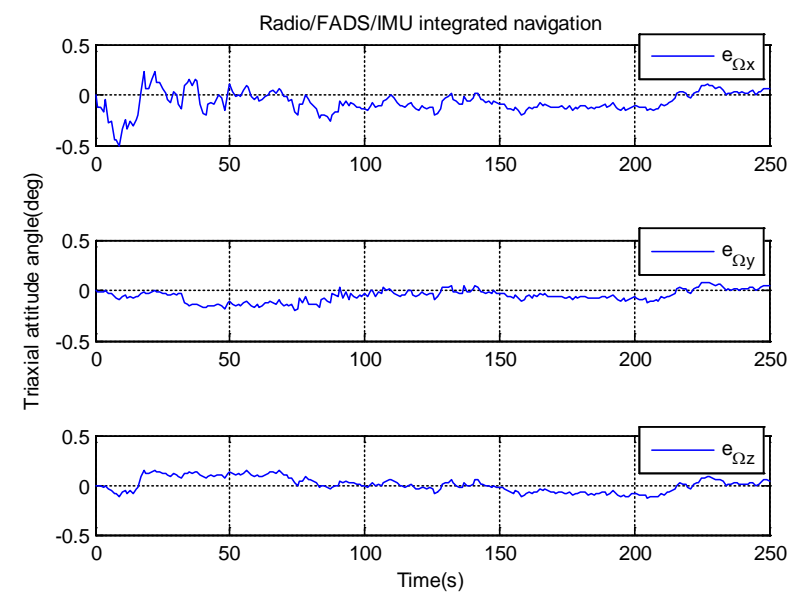

Fig.20. Tri-axial attitude errors from Radio/FADS/IMU integrated navigation.

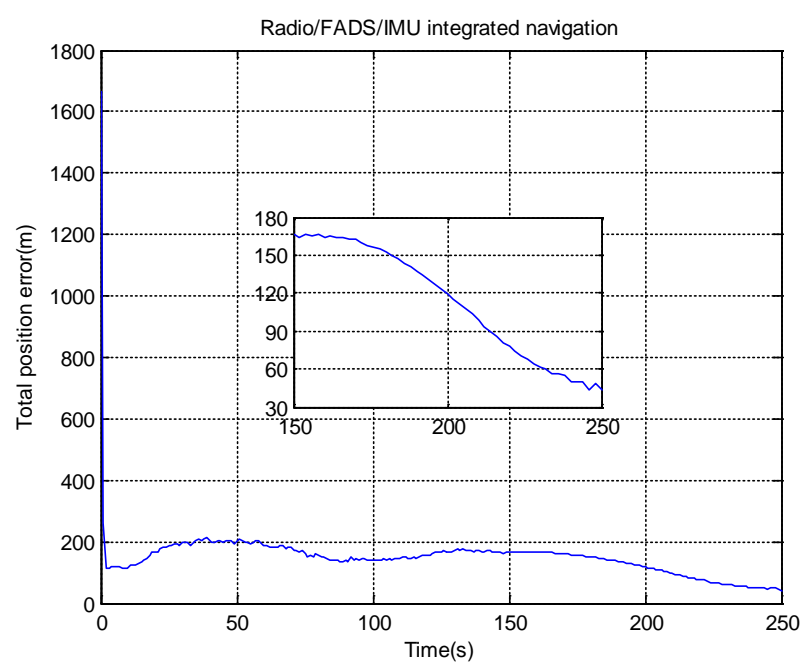

Fig.21. Total position error from Radio/FADS/IMU integrated navigation. 


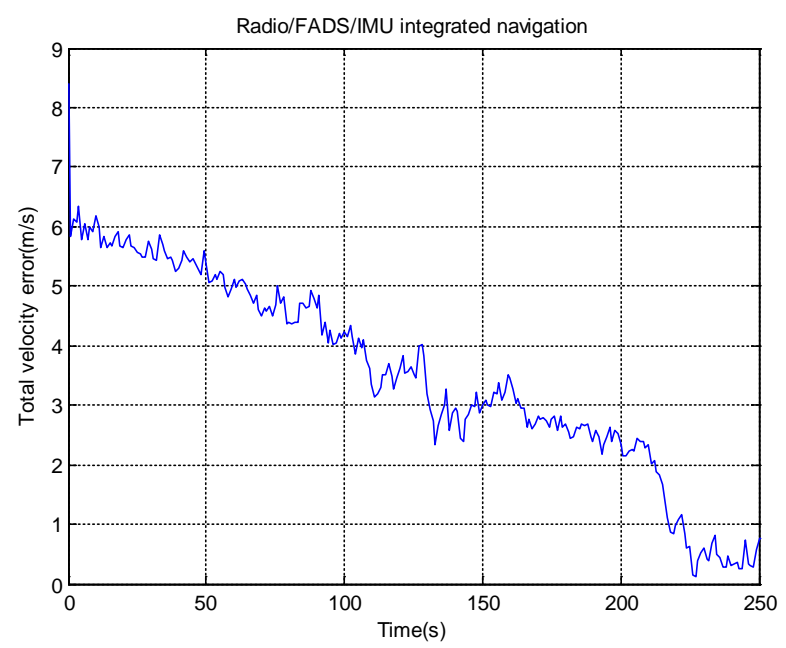

Fig.22. Total velocity error from Radio/FADS/IMU integrated navigation.

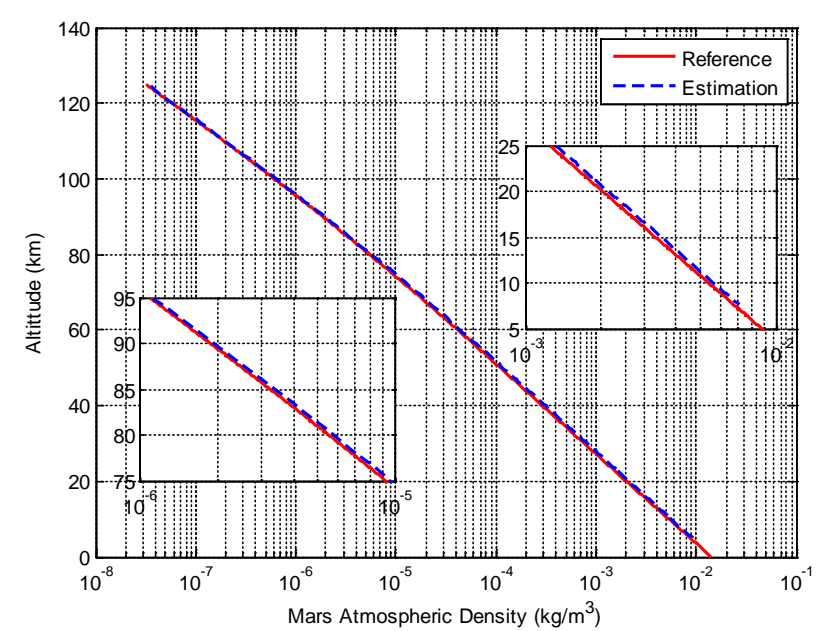

Fig.23. Mars atmospheric density estimation during entry phase.

\section{Conclusion}

Precise navigation is one of the prerequisites for high-precision entry guidance of future Mars missions. In this paper, radio/FADS/IMU integrated navigation scheme for Mars entry has been numerically verified as a candidate solution to support the given Mars orbiting/landing collaborative exploration mission. The acceleration and angular velocity data sensed from IMU, the range and velocity from the entry vehicle to the orbiter obtained from radio measurement, and the dynamic pressure and heating data derived from FADS are used as navigation measurements. Entry state variables are accurately estimated by unscented kalman filter. Simulation cases show that although the state estimation errors from both the one orbiting radio beacon/IMU and the FADS/IMU integrated navigation schemes cannot be well convergent, the radio/FADS/IMU integrated navigation scheme can achieve the position error of $200 \mathrm{~m}$ and the velocity error of $6 \mathrm{~m} / \mathrm{s}$ which is one order of magnitude better than that of the other two 
schemes. And the latter can theoretically meet the navigation accuracy needs of the given China's Mars orbiting/landing collaborative mission case. The number of radio beacons, precision of orbit determination, and measurement accuracy of flush air data sensing system have a significant impact on the integrated navigation performance. These issues are left for the further work.

\section{Acknowledgments}

The work described in this paper was supported by the National Natural Science Foundation of China (Grant No. 11672126, 61673057 and 61273051), the Opening Grant from the Key Laboratory of Space Utilization, Chinese Academy of Sciences (LSU-2016-07-01), Funding of Jiangsu Innovation Program for Graduate Education (Grant No. KYZZ16_0170), the Fundamental Research Funds for the Central Universities, and Funding for Outstanding Doctoral Dissertation in NUAA (Grant No. BCXJ16-10). The first author would like to acknowledge the financial support provided by the China Scholarship Council for his study at the University of Arizona (Grant No. 201706830055).

\section{Appendix A: Coordinate Systems and Transformation Matrixes}

Fig.A.1 depicts the relative geometrical relationship among these coordinate systems. The coordinate systems definition and the coordinate transformation matrixes are defined as follows.

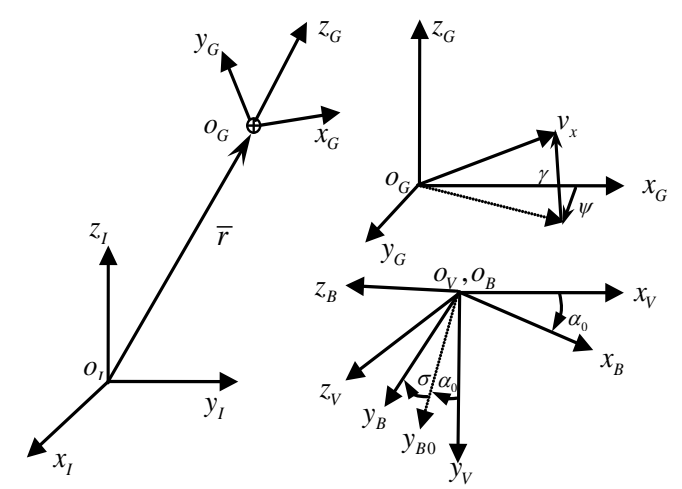

Fig.A.1 Relative geometrical relationship between coordinate systems

\section{(1) Mars centered inertial coordinate system ( $\left.\Sigma^{I}\right)$}

The origin of the Mars centered inertial coordinate system $o_{I}-x_{I} y_{I} z_{I}$ is centered on Mars, and the $z_{I}$ axis is aligned with the rotation axis of the planet, the $x_{I}$ axis is defined to point to toward the vernal equinox. The $y_{t}$ axis completes the right-handed orthogonal coordinate system. The entry vehicle's position, velocity and attitude parameters used for the integrated navigation algorithm are all described in the Mars centered inertial coordinate system. 


\section{(2) Mars centered Mars-fixed coordinate system $\left(\Sigma^{M}\right)$}

The Mars centered Mars-fixed coordinate system $o_{M}-x_{M} y_{M} z_{M}$ is centered and fixed to the Mars and aligns the $z_{M}$ axis is aligned with the rotation axis of the planet, the coordinate system is obtained from the Mars centered inertial coordinate system by a rotation $\Omega$ around $z_{M}$. The $\Omega$ is angular position from the inertial coordinate system and defined as $\Omega=\Omega_{0}+\omega_{M} t$, and $\omega_{M}$ denotes the Mars rotating angular rate.

The coordinate transformation matrix $\boldsymbol{T}_{I}^{M}$ from the $\Sigma^{I}$ to $\Sigma^{M}$ can be obtained as

$$
\boldsymbol{T}_{I}^{M}=\left[\begin{array}{ccc}
\cos \Omega & \sin \Omega & 0 \\
-\sin \Omega & \cos \Omega & 0 \\
0 & 0 & 1
\end{array}\right]
$$

\section{(3) Body-fixed coordinate system $\left(\Sigma^{B}\right)$}

The origin of body-fixed coordinate system $o_{B}-x_{B} y_{B} z_{B}$ lies in the vehicle's mass center, three body axis of symmetry are defined as three coordinate axes $x_{B}, y_{B}, z_{B}$ respectively. The outputs of inertial measurement unit (accelerometer and gyro) are defined in this coordinate system.

The coordinate transformation matrix $\boldsymbol{T}_{I}^{B}$ from the Mars centered inertial coordinate system $\Sigma^{I}$ to the body-fixed coordinate system $\Sigma^{B}$ can be constructed as

$$
\begin{gathered}
\boldsymbol{T}_{I}^{B}=\left[\begin{array}{ccc}
1 & 0 & 0 \\
0 & \cos \varphi & \sin \varphi \\
0 & -\sin \varphi & \cos \varphi
\end{array}\right]\left[\begin{array}{ccc}
\cos \vartheta & 0 & -\sin \vartheta \\
0 & 1 & 0 \\
\sin \vartheta & 0 & \cos \vartheta
\end{array}\right]\left[\begin{array}{ccc}
\cos \phi & \sin \phi & 0 \\
-\sin \phi & \cos \phi & 0 \\
0 & 0 & 1
\end{array}\right] \\
=\left[\begin{array}{ccc}
\cos \vartheta \cos \phi & \cos \vartheta \sin \phi & -\sin \vartheta \\
-\cos \varphi \sin \phi+\sin \varphi \sin \vartheta \cos \phi & \cos \varphi \cos \phi+\sin \varphi \sin \vartheta \sin \phi & \sin \varphi \cos \vartheta \\
\sin \varphi \sin \phi+\cos \varphi \sin \vartheta \cos \phi & -\sin \varphi \cos \phi+\cos \varphi \sin \vartheta \sin \phi & \cos \varphi \cos \vartheta
\end{array}\right]
\end{gathered}
$$

where $\phi, \theta, \varphi$ are the tri-axial attitude angles (i.e. Euler angles).

(4) Velocity coordinate system $\left(\Sigma^{v}\right)$

The origin of velocity coordinate system $o_{V}-x_{V} y_{V} z_{V}$ lies in the vehicle's mass center, the $x_{V}$ axis is aligned with the relative atmospheric velocity, and the $z_{V}$ axis is in local vertical plane and orthogonal to $x_{V}$ axis. The $y_{V}$ axis completes the right-handed orthogonal coordinate system. The aerodynamic lift and drag forces are usually defined in this coordinate system.

The coordinate transformation matrix $\boldsymbol{T}_{V}^{B}$ from the velocity coordinate system $\Sigma^{V}$ to the body-fixed coordinate system $\Sigma^{B}$ can be constructed as 


$$
\boldsymbol{T}_{V}^{B}=\left(\boldsymbol{T}_{B}^{V}\right)^{-1}=\left(\left[\begin{array}{ccc}
1 & 0 & 0 \\
0 & \cos \sigma & \sin \sigma \\
0 & -\sin \sigma & \cos \sigma
\end{array}\right]\left[\begin{array}{ccc}
\cos \left(-\alpha_{0}\right) & 0 & -\sin \left(-\alpha_{0}\right) \\
0 & 1 & 0 \\
\sin \left(-\alpha_{0}\right) & 0 & \cos \left(-\alpha_{0}\right)
\end{array}\right]\right)^{-1}
$$

where $\sigma$ denotes the bank angle and $\alpha_{0}$ denotes the equivalent angle-of-attack.

The coordinate transformation matrix $\boldsymbol{T}_{V}^{I}$ from the velocity coordinate system $\Sigma^{V}$ to the Mars centered inertial coordinate system $\Sigma^{I}$ can be easily obtained

$$
\boldsymbol{T}_{V}^{I}=\left(\boldsymbol{T}_{I}^{B}\right)^{-1} \boldsymbol{T}_{V}^{B}
$$

\section{(5) Geographic coordinate system $\left(\Sigma^{G}\right)$}

The origin of geographic coordinate system $o_{G}-x_{G} y_{G} z_{G}$ lies in the vehicle's mass center, and the three coordinate axes $x_{G}, y_{G}, z_{G}$ are aligned with Mars geographic directions East, North and Up respectively.

The aerodynamic forces and the Mars gravity acceleration is defined in this coordinate system.

The coordinate transformation matrix $\boldsymbol{T}_{M}^{G}$ from the Mars centered Mars-fixed coordinate system $\Sigma^{M}$ to the geographic coordinate system $\Sigma^{G}$ can be constructed as follows

$$
\boldsymbol{T}_{M}^{G}=\left[\begin{array}{ccc}
0 & 0 & 1 \\
0 & 1 & 0 \\
-1 & 0 & 0
\end{array}\right]\left[\begin{array}{ccc}
\cos \lambda \cos \theta & \cos \lambda \sin \theta & \sin \lambda \\
-\sin \theta & \cos \theta & 0 \\
-\sin \lambda \cos \theta & -\sin \lambda \sin \theta & \cos \lambda
\end{array}\right]
$$

where $\theta$ denotes areocentric longitude and $\lambda$ denotes areocentric latitude.

The coordinate transformation matrix $\boldsymbol{T}_{G}^{I}$ from the geographic coordinate system $\Sigma^{G}$ to the Mars centered inertial coordinate system $\Sigma^{I}$ can be constructed as follows

$$
\boldsymbol{T}_{G}^{I}=\left(\boldsymbol{T}_{I}^{M}\right)^{-1}\left(\boldsymbol{T}_{M}^{G}\right)^{-1}
$$

\section{Appendix B: Identification Process of the Flush Air Data Sensing System}

The pressure transducer record the surface pressure at each orifice on the forebody, and the pressure is a function of pressure orifice location, angle of attack, angle of sideslip, Mach number. Explicitly, the surface pressure at the ith orifice is given as

$$
\begin{aligned}
p_{i} & =p_{\infty}+q_{\infty} \cos ^{2} \varsigma_{i}=p_{\infty}+0.5 \rho\|\boldsymbol{v}\|^{2} \cos ^{2} \varsigma_{i} \\
& =p_{t}\left((1-E) \cos ^{2} \varsigma_{i}+E\right)
\end{aligned}
$$

where $p_{i}$ is the measured pressure value of the ith pressure orifice, $p_{t}$ is the total pressure at stagnation, $p_{\infty}$ is the static pressure, $q_{\infty}$ is the dynamic pressure, $E$ is the ratio of static pressure to total pressure, $\varsigma_{i}$ is the incidence angle, it defines as the angle between the direction of the entry velocity and the axis of the ith pressure orifice. 


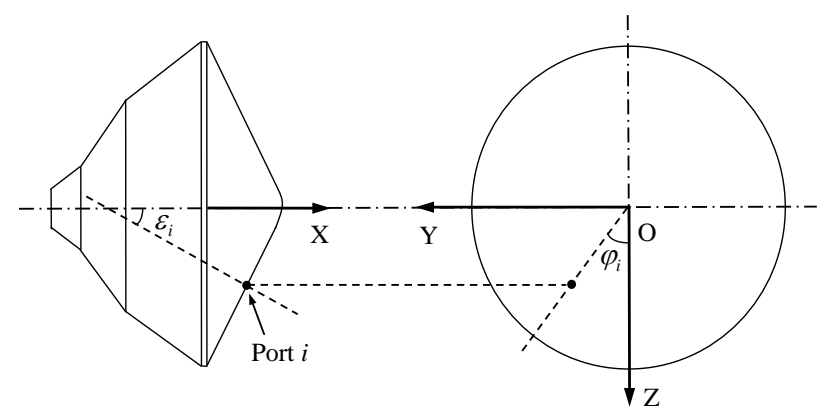

Fig. B.1 Sketch of FADS port geography

The position of the pressure orifice on the aeroshell of the entry vehicle is shown in Fig. B.1, $\varepsilon_{i}$ is the cone angle, and $\varphi_{i}$ is the clock angle of the orifice.

$$
\cos \varsigma_{i}=\cos \alpha \cos \beta \cos \varepsilon_{i}+\sin \beta \sin \varphi_{i} \cos \varepsilon_{i}+\sin \alpha \sin \beta \cos \varphi_{i} \sin \varepsilon_{i}
$$

The pressure radio $E$ is a function of the Mach number

$$
E=\frac{p_{\infty}}{p_{t}}= \begin{cases}\left(\frac{2}{\left(1+c_{\kappa}\right) M a^{2}}\right)^{\frac{c_{\kappa}}{c_{\kappa}-1}}\left(\frac{2 c_{\kappa} M a^{2}-\left(c_{\kappa}-1\right)}{1+c_{\kappa}}\right)^{\frac{1}{c_{*}-1}} & , M a>1 \\ \left(1+\frac{c_{\kappa}-1}{2} M a^{2}\right)^{\frac{c_{\kappa}}{c_{k}-1}} & , M a \leq 1\end{cases}
$$

where $c_{\kappa}$ is the isentropic expansion coefficient (assumed to be 1.335), and Ma is the Mach number defined as the ratio of the entry velocity to the sound velocity, i.e. $M a=\|\boldsymbol{v}\| / v_{S}$. The speed of sound $v_{S}$ is estimated by $[26,28]$

$$
v_{S}=15.74 \sqrt{242.15-0.998 h}
$$

where $h$ is the flight altitude of the entry vehicle.

Besides, heat transducers sense the heating rate $\mathcal{F}^{\&}$ at the stagnation point of the entry vehicle during the trim angle of attack state.

\section{Appendix C: Influence of Wind during Mars Entry Phase}

The measurement of the dynamic pressure is inevitably affected by wind speed.

$$
\Delta q=\frac{0.5 \rho\left(\|\boldsymbol{v}\|+\left\|\boldsymbol{v}_{\text {wind }}\right\|\right)^{2}-0.5 \rho\|\boldsymbol{v}\|^{2}}{0.5 \rho\left(\|\boldsymbol{v}\|+\left\|\boldsymbol{v}_{\text {wind }}\right\|\right)^{2}}
$$

In the analysis, the average wind speed is assumed to be $\left\|\boldsymbol{v}_{\text {wind }}\right\|=10 \mathrm{~m} / \mathrm{s}$, considering the worst case that the wind is on the opposite direction of the entry vehicle velocity. For the Mars hypersonic entry, the nominal velocity $\|v\|$ is about $6000 \mathrm{~m} / \mathrm{s}$ to $450 \mathrm{~m} / \mathrm{s}$. Then, deviation percentage of the dynamic pressure measurement caused by the wind 
speed can be evaluated, that is about $0.33 \%$ to $4.49 \%$.

Therefore, the effect of the wind speed on dynamic pressure measurement is treated as dynamic pressure measurement noise accordingly in this paper.

\section{References}

[1] Ingoldby, R. N. Guidance and control system design of the Viking planetary lander. Journal of Guidance, Control, and Dynamics, 1, 3 (1978), 189-196.

[2] Genova, A., Goossens, S., Lemoine, F. G., Mazarico, E., Neumann, G. A., Smith, D. E., and Zuber, M. T. Seasonal and static gravity field of Mars from MGS, Mars odyssey and MRO radio science. Icarus, 272 (2016), 228-245.

[3] Srivastava, V. K., Kumar, J., Kulshrestha, S., Kushvah, B. S., Bhaskar, M. K., Somesh, S., Roopa, M. V., and Ramakrishna, B. N. Eclipse modeling for the Mars orbiter mission. Advances in Space Research, 56, 4 (2015), 671-679.

[4] Spencer, D. A., Blanchard, R. C., Braun, R. D., Kallemeyn, P. H., and Thurman, S. W. Mars pathfinder entry, descent, and landing reconstruction. Journal of Spacecraft and Rockets, 36, 3 (1999), 357-366.

[5] Desai, P. N., Lee, W. J., and Steltzner, A. Entry, descent, and landing scenario for the Mars exploration rover mission. The Journal of the Astronautical Sciences, 55, 4 (2007), 421-430.

[6] Kornfeld, R. P., Garcia, M. D., Craig, L. E., Butman, S., and Signori, G. M. Entry, descent, and landing communications for the 2007 phoenix Mars lander. Journal of Spacecraft and Rockets, 45, 3 (2008), 534-547.

[7] Williams, J. L., Menon, P. R., and Demcak, S. W. Mars reconnaissance orbiter navigation strategy for Mars science laboratory entry, descent and landing telecommunication relay support. Presented at the AIAA/AAS Astrodynamics Specialist Conference, Minneapolis, Minnesota, AIAA 2012-4747, Aug. 2012.

[8] Renault, H., Sergent, N., Chevallier, M., Kutrowski, N., Bacchetta, A., Temperanza D. ExoMars 2016, orbiter module bus a GNC development update. CEAS Space Journal, 7, 2 (2015), 105-118.

[9] Jiang, X. Q., Li, S. Enabling technologies for Chinese Mars lander guidance system. Acta Astronautica, 133, (2017), 375-386.

[10] Steinfeldt, B. A., Grant, M. J., Matz, D. A., Braun, R. D., and Barton, G. H. Guidance, navigation, and control system performance trades for Mars pinpoint landing. Journal of Spacecraft and Rockets, 47, 1 (2010), 188-198.

[11] Li, S., and Jiang X. Q. Review and prospect of guidance and control for Mars atmospheric entry. Progress in Aerospace Sciences, 69 (2014), 40-57.

[12] Paschall, S. C. Mars entry navigation performance analysis using monte carlo techniques. Doctoral Dissertation, Massachusetts Institute of Technology, 2004.

[13] Cui, P. Y., Yu, Z. S., and Zhu, S. Y. Research progress and prospect of autonomous navigation techniques for Mars entry phase. 
Journal of Astronautics, 34, 4 (2013), 447-456.

[14] Li, S., and Peng, Y. M. Radio beacons/IMU integrated navigation for Mars entry. Advances in Space Research, 47, 1 (2011), 1265-1279.

[15] Li, S., Jiang, X. Q., and Liu, Y. F. Innovative Mars entry integrated navigation using modified multiple model adaptive estimation. Aerospace Science and Technology, 39 (2014), 403-413.

[16] Li, S., Jiang, X. Q., and Liu, Y. F. High-precision Mars entry integrated navigation under large uncertainties. Journal of Navigation, 67, 2 (2014), 327-342.

[17] Lou, T. S., Fu, H. M., Zhang, Y. B., and Wang, Z. H. Consider unobservable uncertain parameters using radio beacon navigation during Mars entry. Advances in Space Research, 55, 4 (2015), 1038-1050.

[18] Yu, Z. S., Cui, P. Y., and Zhu, S. Y. On the observability of Mars entry navigation using radiometric measurements. Advances in Space Research, 54, 8 (2014), 1513-1524.

[19] Wang, X., and Xia, Y. Q. Navigation strategy with the spacecraft communications blackout for Mars entry. Advances in Space Research, 55, 4 (2015), 1264-1277.

[20] Wang, L. S., and Xia, Y. Q. Observability analysis of Mars entry integrated navigation. Advances in Space Research, 56, 5 (2015), 952-963.

[21] Yu, Z. S., Cui, P. Y., and Zhu, S. Y. Observability-based beacon configuration optimization for Mars entry navigation. Journal of Guidance, Control, and Dynamics, 38, 4 (2014), 643-650.

[22] Yu, Z. S., Zhu, S. Y., and Cui, P. Y. Orbit optimization of Mars orbiters for entry navigation: from an observability point of view. Acta Astronautica, 111 (2015), 136-145.

[23] Wang, P., Jin, X., Zhang, W. Research on the solving accuracy for the FADS system applied to the vehicle with blunt fore-bodies. Mechanics in Engineering, 2016, 38(3): 255-261.

[24] Wang, P, Jin, X., Zhang, W. Pressure ports configuration for the flush air data sensing system. Mechanics in Engineering, in press, 2017.

[25] Zhang, M. Research on flush air data sensing system in hypersonic flight. Master Dissertation, Nanjing University of Aeronautics and Astronautics, 2014.

[26] Cheatwood, F. M., Bose, D., Karlgaard, C. D., Kuhl, C. A., Santos, J. A., and Wright, M. J. Mars science laboratory (MSL) entry, descent, and landing instrumentation (MEDLI): complete flight data set. NASA Report, NASA/TM-2014-218533, 2014.

[27] Deng, J., Gao, A., Zong, H., and Cui, P. An innovative navigation scheme for Mars entry using dynamic pressure measurement. Advances in Space Research, 60, 10 (2017), 2319-2331.

[28] Dutta, S. Statistical methods for reconstruction of entry, descent, and landing performance with application to vehicle design. 
Ph.D. Dissertation, Georgia Institute of Technology, 2013.

[29] Benito Manrique, J. Advances in spacecraft atmospheric entry guidance. Ph.D. Dissertation, University of California, 2010.

[30] Yang, L., Hou, Y., Zuo, G., Liu, Y., and Guo, B. Aerodynamic characteristics measurement of Mars vehicles during entry flight. Chinese Journal of Theoretical and Applied Mechanics, 47, 1 (2015), 8-14.

[31] Benito, J., Mease, K. D. Reachable and controllable sets for planetary entry and landing. Journal of Guidance, Control and Dynamics, 33, 3 (2012), 641-654.

[32] Mendeck, G. F., and McGrew, L. C. Entry guidance design and postflight performance for 2011 Mars science laboratory mission. Journal of Spacecraft and Rockets, 51, 4 (2014), 1094-1105. 